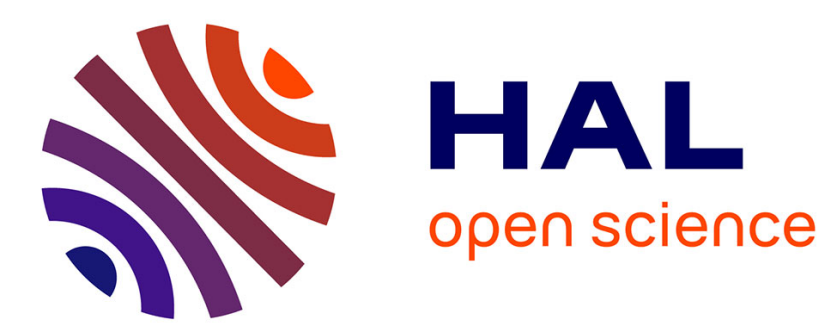

\title{
Représentation analytique des surfaces d'aimantation en vue de la simulation des machines électriques
}

\author{
H.A. Anvari, A. Xhuvani, J. Faucher
}

\section{To cite this version:}

H.A. Anvari, A. Xhuvani, J. Faucher. Représentation analytique des surfaces d'aimantation en vue de la simulation des machines électriques. Revue de Physique Appliquée, 1989, 24 (8), pp.803-819. 10.1051/rphysap:01989002408080300 . jpa-00246103

\section{HAL Id: jpa-00246103 https://hal.science/jpa-00246103}

Submitted on 1 Jan 1989

HAL is a multi-disciplinary open access archive for the deposit and dissemination of scientific research documents, whether they are published or not. The documents may come from teaching and research institutions in France or abroad, or from public or private research centers.
L'archive ouverte pluridisciplinaire HAL, est destinée au dépôt et à la diffusion de documents scientifiques de niveau recherche, publiés ou non, émanant des établissements d'enseignement et de recherche français ou étrangers, des laboratoires publics ou privés. 


\title{
Représentation analytique des surfaces d'aimantation en vue de la simulation des machines électriques
}

\author{
H. A. Anvari, A. Xhuvani et J. Faucher \\ Laboratoire d'Electrotechnique et d'Electronique Industrielle, U. A. au CNRS n 847,2 rue Camichel, \\ 31071 Toulouse Cedex, France
}

(Reçu le 27 octobre 1988, révisé le 3 mars 1989, accepté le 10 mai 1989)

\begin{abstract}
Résumé. - Les auteurs présentent la forme analytique des surfaces d'aimantation dans une machine à courant alternatif saturée. L'utilisation de ces surfaces dans un modèle qui tient compte de l'effet croisé de saturation permet d'aboutir à des résultats précis pour l'étude du comportement des machines.
\end{abstract}

\begin{abstract}
The authors present an analytical representation of magnetisation surfaces for saturated a.c. machines. A more precise model for studying machine behaviours based on utilisation of these surfaces which takes into consideration the cross coupling magnetisation effect is then developed.
\end{abstract}

\section{Liste des principaux symboles}

$R \quad:$ résistance d'enroulement (ohm)

$\lambda_{\mathrm{d}}, \lambda_{\mathrm{q}}$ : flux commun suivant l'axe $\mathrm{d}, \mathrm{q}(\mathrm{Wb})$

I $\quad$ : courant (A)

$V \quad:$ tension (V)

$\phi \quad:$ flux total d'enroulement $(\mathrm{Wb})$

I $\quad$ : inductance de fuites $(\mathrm{H})$

$L_{\mathrm{md}}, L_{\mathrm{mq}}$ : inductance statique suivant l'axe d, q (H)

$\mathfrak{L}_{\mathrm{md}}, \mathfrak{L}_{\mathrm{mq}}:$ inductance dynamique suivant l'axe $\mathrm{d}, \mathrm{q}$ (H)

$L_{\mathrm{qd}}, L_{\mathrm{dq}}$ : coefficients équivalents à l'inductance statique dus à l'effet croisé $(\mathrm{H})$

$\mathfrak{L}_{\mathrm{qd}}, \mathfrak{L}_{\mathrm{dq}}$ : coefficients équivalents à l'inductance dynamique dus à l'effet croisé $(\mathrm{H})$

$\varepsilon \quad$ : fonction d'erreur

\section{Liste des indices}

$\mathrm{d}, \mathrm{q} \quad$ : relatif à l'axe direct et quadrature

sd, sq : relatif à l'enroulement équivalent statorique suivant l'axe $\mathrm{d}, \mathrm{q}$

$\mathrm{kd}, \mathrm{kq}$ : relatif à l'enroulement amortisseur suivant l'axe d, $\mathrm{q}$

f : relatif à l'enroulement d'excitation

\section{Introduction.}

Le modèle traditionnel de la décomposition vectorielle spatiale et temporelle tant des champs magnétiques que des courants dans les machines électriques à courants alternatifs est extrêmement utilisé. Ce modèle permet de simuler de façon simple le comportement en régime transitoire de ces machines. Son utilisation en régime de saturation magnétique pose cependant quelques problèmes sur lesquels il convient de se pencher.

Il est indispensable de connaître les caractéristiques d'aimantation sur les deux axes $d$ et $q$. La courbe de magnétisation sur l'axe longitudinal (axe d) est fournie par' les caractéristiques à vide et en court-circuit, alors que la détermination de cette courbe sur l'axe transversal (axe q) est plus délicate et les normes proposent plusieurs méthodes : soit un essai à excitation négative, soit un essai à faible glissement.

L'étude plus détaillée [1-16] de la saturation des machines montre qu'il existe, à cause de l'effet croisé de saturation, non une courbe unique d'aimantation sur chaque axe mais une famille de courbes. Chaque courbe représente alors la variation du flux en fonction du courant sur un axe, le courant suivant l'autre axe étant maintenu constant.

Une fois les courbes expérimentalement obtenues, il faut les présenter analytiquement afin de les exploiter ultérieurement dans la phase de simulation. On peut se demander si ce genre d'exercice est encore bien nécessaire car, grâce aux ordinateurs et aux logiciels il serait aussi possible de faire des interpolations à partir d'un tableau de données expérimentales. Cela est vrai dans le cas des courbes classiques d'aimantation, mais lorsqu'il s'agit d'intro- 
duire l'effet croisé de saturation, les résultats expérimentaux ne sont pas disponibles dans toute la plage de variation des flux et des courants. Ceci nous oblige à avoir recours à des solutions analytiques.

Depuis longtemps beaucoup de travaux ont été effectués [17] qui concernent la représentation analytique des courbes d'aimantation des machines électriques. La prise en compte de l'effet croisé de saturation dans l'étude du comportement des machines à courant alternatif est assez récente et la représentation des familles de courbes suivant les deux axes est peu développée dans les ouvrages scientifiques et techniques dans le cadre de cette hypothèse. La notion de surface d'aimantation à notre connaissance est originale et l'utilisation de ces surfaces dans le modèle mathématique des machines électriques nous conduit à un modèle plus réaliste dans l'étude du comportement de ces machines aussi bien en régime permanent qu'en régime transitoire.

Afin de développer ces différents points, une étude sur l'effet croisé de saturation est présentée et appliquée à la modélisation de la machine synchrone. Ensuite, à partir de cette étude préliminaire, les auteurs proposent différentes formes analytiques des surfaces d'aimantation ainsi que les méthodes d'identification des paramètres de ces surfaces.

Enfin un exemple d'application est traité qui concerne une machine à réluctance fortement saturée.

\section{L'effet croisé de saturation, modèle de Park généralisé.}

La méthode de transformation de Park qui permet, par un changement de référentiel, de passer du système statorique au système rotorique avec élimination au passage de certaines variables, représente la meilleure méthode de calcul pour les machines synchrones. Cependant, l'utilisation de cette méthode en cas de saturation, est extrêmement délicate et nécessite quelques précautions et modifications.

Reprenons tout d'abord deux hypothèses qui permettent de définir cette transformation : la répartition sinusoïdale des f.m.m. le long de l'entrefer et la répartition sinusoïdale de l'induction dans l'entrefer.

Si la première hypothèse ne présente pas de problème dans la mesure où la machine est construite de façon appropriée, la deuxième est plus difficile à satisfaire car il est bien évident que dans le cas de saturation, les effets ne sont pas proportionnels aux causes. On ne peut donc pas, en toute rigueur, considérer qu'une variation spatiale sinusoïdale de courant va produire une variation spatiale sinusoïdale de l'induction. En fait, en régime de saturation, on voit apparaître des harmoniques impairs d'espace dans la variation de $B$ le long de l'entrefer. Il faut cependant constater que l'induction dans l'entrefer n'intervient que par intégration spatiale pour déterminer le flux à travers les enroulements et que les harmoniques d'espace conduisent souvent à des harmoniques de temps de faible amplitude.

Pour établir des transformations et écrire des équations de machines suivant les axes $d$ et $q$, l'hypothèse d'une répartition sinusoïdale des courants permet la détermination de $I_{\mathrm{md}}$ et $I_{\mathrm{mq}}$. La décomposition du flux total en deux flux $\lambda_{d}$ et $\lambda_{\mathrm{q}}$ ayant été rendue possible en négligeant les harmoniques d'espace.

En admettant qu'il n'y a aucun couplage magnétique entre les enroulements fictifs disposés en quadrature, les flux $\lambda_{\mathrm{d}}$ et $\lambda_{\mathrm{q}}$ peuvent être calculés à partir des courants $I_{\mathrm{md}}$ et $I_{\mathrm{mq}}$ et des courbes d'aimantation au moyen des relations :

$$
\begin{aligned}
& \lambda_{\mathrm{d}}=\lambda_{\mathrm{d}}\left(I_{\mathrm{md}}\right) \\
& \lambda_{\mathrm{q}}=\lambda_{\mathrm{q}}\left(I_{\mathrm{mq}}\right)
\end{aligned}
$$

$I_{\mathrm{md}}$ et $I_{\mathrm{mq}}$ sont des courants effectifs sur les axes d et q soit

$$
\begin{gathered}
I_{\mathrm{md}}=I_{\mathrm{sd}}+I_{\mathrm{f}}+I_{\mathrm{kd}} \\
I_{\mathrm{mq}}=I_{\mathrm{sq}}+I_{\mathrm{sq}} .
\end{gathered}
$$

Cette façon de faire ne peut, en toute rigueur être valable que si le système est linéaire. Dans le cas où $\lambda_{\mathrm{d}}$ et $\lambda_{\mathrm{q}}$ sont non linéaires, cette méthode mène à des erreurs et conduit à une surévaluation du flux. L'expérience a montré en effet qu'il existe à partir d'un seuil de saturation, un couplage magnétique entre les enroulements sur l'axe d et les enroulements sur l'axe q appelé effet croisé de saturation. Afin d'avoir une plus grande précision dans les résultats de calcul, il faut considérer l'influence du courant dans un axe sur le flux dans l'autre axe. Cette influence croisée de la saturation peut avoir une incidence relativement importante sur la caractéristique des machines. Une décomposition de flux qui prendrait en compte cette influence croisée de saturation conduit à :

$$
\begin{aligned}
& \lambda_{\mathrm{d}}=\lambda_{\mathrm{d}}\left(I_{\mathrm{md}}, I_{\mathrm{mq}}\right) \\
& \lambda_{\mathrm{q}}=\lambda_{\mathrm{q}}\left(I_{\mathrm{mq}}, I_{\mathrm{md}}\right)
\end{aligned}
$$

à savoir, connaissant $I_{\mathrm{md}}$ et $I_{\mathrm{mq}}$ et une famille de courbes d'aimantation sur chaque axe $d$ et $q$, on détermine $\lambda_{\mathrm{d}}$ et $\lambda_{\mathrm{q}}$. En revanche, étant donné la non-linéarité de cette fonction, la détermination des courants à partir des flux n'est possible que par une procédure itérative. (Nous allons voir plus loin qu'au lieu de ces familles de courbes d'aimantation magnétique, on peut utiliser des surfaces d'aimantation.)

Si on considère $\lambda_{\mathrm{d}}, I_{\mathrm{md}}, I_{\mathrm{mq}}$ comme les trois axes d'un système de coordonnées $X=I_{\mathrm{md}}, Y=I_{\mathrm{mq}}$, $Z=\lambda_{\mathrm{d}}, \lambda_{\mathrm{d}}$ présente une surface définie par la relation $Z=f(x, y)$. De la même manière en consi- 
dérant $\lambda_{\mathrm{q}}, I_{\mathrm{mq}}$ et $I_{\mathrm{md}}$ les trois axes d'un autre système de coordonnées $x=I_{\mathrm{mq}} y=I_{\mathrm{md}}$ et $z=\lambda_{\mathrm{q}}$, une deuxième surface est définie.

2.1 Modélisation DE LA MACHINE. - Considérons le modèle de la machine synchrone excitée avec circuits amortisseurs qui est présenté à la figure 1 .

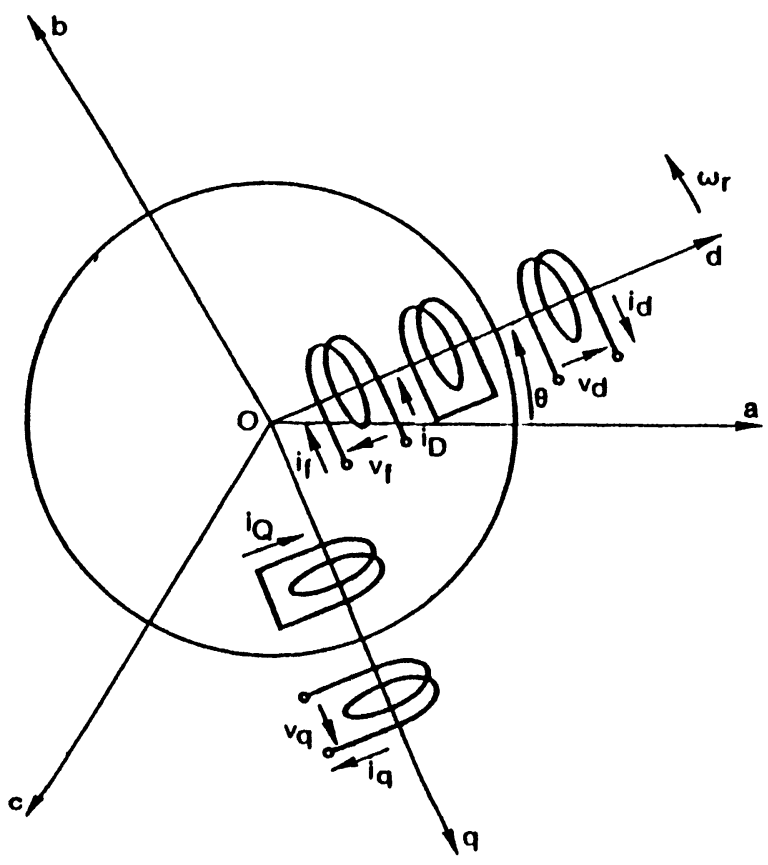

Fig. 1. - Représentation du modèle de la machine synchrone.

[Model representation of synchronous machine.]

L'équation différentielle matricielle de fonctionnement de cette machine suivant les axes $d$ et $q$ est :

$$
|V|=|R| \cdot|I|+|S| \cdot|\Phi|+|\dot{\Phi}|
$$

dans laquelle :

$|V|=$ matrice colonne des tensions

$$
=\left|V_{\mathrm{sd}}, V_{\mathrm{kd}}, V_{\mathrm{f}}, V_{\mathrm{sq}}, V_{\mathrm{kq}}\right|^{\mathrm{t}}
$$

$|I|=$ matrice colonne des courants

$$
=\left|I_{\mathrm{sd}}, I_{\mathrm{kd}}, I_{\mathrm{f}}, I_{\mathrm{sq}}, I_{\mathrm{kq}}\right|^{\mathrm{t}}
$$

$|R|=$ matrice diagonale des résistances

$$
=\operatorname{diag} .\left|R_{\mathrm{sd}}, R_{\mathrm{kd}}, R_{\mathrm{f}}, R_{\mathrm{sq}}, R_{\mathrm{kq}}\right|
$$

$|\Phi|=$ matrice colonne des flux

$$
=\left|\Phi_{\text {sd }}, \Phi_{\mathrm{kd}}, \Phi_{\mathrm{f}}, \Phi_{\mathrm{sq}}, \Phi_{\mathrm{kq}}\right|^{\mathrm{t}}
$$

$|S|=$ matrice «tension de rotation » avec tous les éléments égaux à zéro sauf $S_{14}=-S_{41}=$ - $\omega$, où $\omega$ est la vitesse de synchronisme.

Les expressions donnant les flux créés dans les différents enroulements s'expriment en fonction des courants circulant dans ceux-ci en utilisant la relation matricielle :

$$
|\Phi|=|\ell| \cdot|I|+|\lambda|
$$

dans laquelle :

$|\ell|=$ matrice diagonale des inductances de fuite $=\left|I_{\mathrm{sd}}, I_{\mathrm{kd}}, I_{\mathrm{f}}, I_{\mathrm{sq}}, I_{k q}\right|$

$|\lambda|=\left|\lambda_{\mathrm{d}}, \lambda_{\mathrm{d}}, \lambda_{\mathrm{d}}, \lambda_{\mathrm{q}}, \lambda_{\mathrm{q}}\right|^{\mathrm{t}^{\mathrm{t}}}$

En considérant l'effet croisé de saturation, les inductances sur l'axe $\mathrm{d}$ et $\mathrm{q}$ dépendent alors chacune des courants $I_{\mathrm{md}}$ et $I_{\mathrm{mq}}$.

2.2 CAlCul DES induCTANCES. - La détermination des inductances sur l'axe $d$ et sur l'axe $q$, soit expérimentalement soit par un calcul du champ, permet pour une structure donnée, de connaître précisément l'importance de l'effet croisé de saturation.

Les résultats de mesure donnent en principe des caractéristiques $L_{\mathrm{md}}\left(I_{\mathrm{md}}, I_{\mathrm{mq}}\right)$ et $L_{\mathrm{mq}}\left(I_{\mathrm{mq}}, I_{\mathrm{md}}\right)$, qui après les mesures des inductances de fuite peuvent être exploitées pour tracer les courbes d'aimantation de la machine sur les deux axes.

En considérant que les inductances $L_{\mathrm{md}}$ et $L_{\mathrm{mq}}$ sont fonctions des deux courants $I_{\mathrm{md}}$ et $I_{\mathrm{mq}}$, on peut écrire :

$$
\begin{aligned}
& \lambda_{\mathrm{d}}=L_{\mathrm{md}}\left(I_{\mathrm{md}}, I_{\mathrm{mq}}\right) \cdot I_{\mathrm{md}} \\
& \lambda_{\mathrm{q}}=L_{\mathrm{mq}}\left(I_{\mathrm{mq}}, I_{\mathrm{md}}\right) \cdot I_{\mathrm{mq}} .
\end{aligned}
$$

On peut considérer qu'il existe une inductance statorique pour chaque axe qui dépend uniquement du courant sur le même axe et un effet croisé qui se traduit par le produit d'un paramètre $L_{\mathrm{dq}}$ ou $L_{\text {qd }}$ par le courant sur l'autre axe.

En séparant les inductances dues à chaque courant et en introduisant les coefficients d'inductances statiques $L_{\mathrm{md}}, L_{\mathrm{mq}}$ et les coefficients dus à l'effet croisé, on a alors :

$$
\begin{aligned}
& \lambda_{\mathrm{d}}=L_{\mathrm{md}} \cdot I_{\mathrm{md}}+L_{\mathrm{qd}} \cdot I_{\mathrm{mq}} \\
& \lambda_{\mathrm{d}}=L_{\mathrm{mq}} \cdot I_{\mathrm{mq}}+L_{\mathrm{dq}} \cdot I_{\mathrm{md}} .
\end{aligned}
$$

Ce qui peut se traduire par :

$$
|\Phi|=|C| \cdot|I| \text {. }
$$

$C$ étant une matrice des inductances qui a pour expression :

$|C|=$

$$
\left|\begin{array}{ccccc}
I_{\mathrm{sd}}+L_{\mathrm{md}} & L_{\mathrm{md}} & L_{\mathrm{md}} & L_{\mathrm{qd}} & L_{\mathrm{qd}} \\
L_{\mathrm{md}} & I_{\mathrm{kd}}+L_{\mathrm{md}} & L_{\mathrm{md}} & L_{\mathrm{qd}} & L_{\mathrm{qd}} \\
L_{\mathrm{md}} & L_{\mathrm{md}} & I_{\mathrm{f}}+L_{\mathrm{md}} & L_{\mathrm{qd}} & L_{\mathrm{qd}} \\
L_{\mathrm{dq}} & L_{\mathrm{dq}} & L_{\mathrm{dq}} & I_{\mathrm{sq}}+L_{\mathrm{mq}} & L_{\mathrm{mq}} \\
L_{\mathrm{dq}} & L_{\mathrm{dq}} & L_{\mathrm{dq}} & L_{\mathrm{mq}} & I_{\mathrm{qd}}+L_{\mathrm{mq}}
\end{array}\right|
$$

On obtient :

$$
|\dot{\Phi}|=|D| \cdot|\dot{I}|
$$

$D$ étant la matrice des inductances dynamiques de la forme suivante : 
$|D|=$

$$
=\left|\begin{array}{ccccr}
I_{\mathrm{sd}}+\mathfrak{L}_{\mathrm{md}} & \mathfrak{L}_{\mathrm{md}} & \mathfrak{L}_{\mathrm{md}} & \mathfrak{L}_{\mathrm{qd}} & \mathfrak{L}_{\mathrm{qd}} \\
\mathfrak{L}_{\mathrm{md}} & I_{\mathrm{kd}}+\mathfrak{L}_{\mathrm{md}} & \mathcal{L}_{\mathrm{md}} & \mathfrak{L}_{\mathrm{qd}} & \mathfrak{L}_{\mathrm{qd}} \\
\mathfrak{L}_{\mathrm{md}} & \mathcal{L}_{\mathrm{md}} & I_{\mathrm{f}}+\mathfrak{L}_{\mathrm{md}} & \mathfrak{L}_{\mathrm{qd}} & \mathfrak{L}_{\mathrm{qd}} \\
\mathfrak{L}_{\mathrm{dq}} & \mathfrak{L}_{\mathrm{dq}} & \mathfrak{L}_{\mathrm{dq}} & I_{\mathrm{sq}}+\mathfrak{L}_{\mathrm{mq}} & \mathfrak{L}_{\mathrm{mq}} \\
\mathfrak{L}_{\mathrm{dq}} & \mathfrak{L}_{\mathrm{dq}} & \mathfrak{L}_{\mathrm{dq}} & \mathfrak{L}_{\mathrm{mq}} & I_{\mathrm{kq}}+\mathfrak{L}_{\mathrm{mq}}
\end{array}\right|
$$

dans laquelle $\mathfrak{L}_{\mathrm{md}}=\partial \lambda_{\mathrm{d}} / \partial I_{\mathrm{md}}$ et $\mathfrak{L}_{\mathrm{mq}}=\partial \lambda_{\mathrm{q}} / \partial I_{\mathrm{mq}}$ sont les inductances dynamiques respectivement pour les axes $d$ et $q$ et $\mathfrak{L}_{\mathrm{qd}}=\partial \lambda_{\mathrm{d}} / \partial I_{\mathrm{mq}}$ et $\mathfrak{L}_{\mathrm{dq}}=$ $\partial \lambda_{q} / \partial I_{\mathrm{md}}$ sont des termes dynamiques dus à l'effet croisé de saturation.

L'équation différentielle du modèle de Park généralisé en prenant $I$ comme vecteur d'état devient alors :

$$
|V|=(|R|+|S| \cdot|C|) \cdot|I|+|D| \cdot|\dot{I}| .
$$

Dans un programme de simulation numérique cette équation peut être résolue pas à pas en utilisant la méthode de Runge-Kutta et l'évolution des grandeurs essentielles peut ainsi être obtenue en fonction du temps. Une fois connue l'évolution dans le temps des variables sur les axes, il suffit d'utiliser une transformation inverse $\mathrm{d}, \mathrm{q}$ pour trouver l'évolution des variables réelles.

2.3 PRINCIPE DE LA MESURE DES INDUCTANCES. Afin de vérifier l'existence de l'effet croisé de saturation une série de mesures a été effectuée sur une machine à réluctance variable [14]. D'autres expériences ont aussi été pratiquées sur une machine synchrone à excitation [15].

Le principe de mesure est basé sur la méthode d'intégration des flux (la méthode de Jones). Le montage présenté dans la figure 1 bis permet la mesure de $L_{\text {sd }}$ et $L_{\mathrm{sq}}$ en fonction du courant $I_{0}$ et de l'angle $\psi . \psi$ étant l'angle entre la f.m.m. résultante et l'axe direct. Connaissant les relations reliant $I_{0}, \psi$ et $I_{\mathrm{md}}, I_{\mathrm{mq}}$ et en mesurant les inductances de fuites $I_{\text {sd }}$ et $I_{\text {sq }}$, les courbes d'aimantation $\lambda_{\mathrm{d}}\left(I_{\mathrm{md}}, I_{\mathrm{mq}}\right)$ sur l'axe direct et $\lambda_{\mathrm{q}}\left(I_{\mathrm{mq}}, I_{\mathrm{md}}\right)$ sur l'axe transversal peuvent être obtenues. Par la suite la partie non-linéaire de ces courbes à l'origine sera linéarisée car le point de fonctionnement de la machine ne se trouve presque jamais dans cette zone.

\section{Représentation analytique des surfaces d'aimanta- tion d'une machine électrique.}

3.1 REPRÉSENTATION ANALYTIQUE DES COURBES D'AIMANTATION SUR L'AXE $d$ ET q. - Après avoir relevé expérimentalement les courbes de $\lambda_{\mathrm{d}}\left(I_{\mathrm{md}}\right)$ et $\lambda_{\mathrm{q}}\left(I_{\mathrm{mq}}\right)$ d'une machine synchrone, il est utile de les représenter dans la zone de fonctionnement au moyen de formules approchées.

On peut évidemment donner les courbes $\lambda_{\mathrm{d}}\left(I_{\mathrm{md}}\right)$ et $\lambda_{\mathrm{q}}\left(I_{\mathrm{mq}}\right)$ au calculateur sous forme de tableaux de valeurs et les exploiter par interpolations linéaires. Cette méthode bien qu'elle soit simple et facile à utiliser, cause des difficultés de calcul aux points d'intersection des deux droites successives et lors des procédures itératives peut amener à l'instabilité des résultats.

Différentes fonctions ont été proposées afin de représenter analytiquement les courbes d'aimantation magnétique (4). Compte tenu de l'allure différente des courbes réelles d'aimantation sur l'axe $d$ et $\mathrm{q}$, les fonctions proposées approximant ces courbes sont en principe différentes. Pour l'axe d le choix s'est orienté vers les fonctions hyperboliques et exponentielles suivantes :

$$
\begin{gathered}
\lambda(I)=\frac{a_{1} I}{b_{0}+b_{1} I} \\
\lambda(I)=a_{1}\left(1-\mathrm{e}^{\frac{-I}{b_{1}}}\right) \\
\lambda(I)=a_{1}\left(1-\mathrm{e}^{\frac{-I}{b_{1}}}\right)+a_{2} I \\
\lambda(I)=\frac{a_{1} \cdot I+a_{2} \cdot I^{2}+\cdots+a_{n} \cdot I^{n}}{1+b_{1} \cdot I+b_{2} \cdot I^{2}+\cdots+b_{n} \cdot I^{n}} \\
\lambda(I)=\frac{a_{0}+a_{1} \cdot I+a_{2} \cdot I^{2}+\cdots a_{n} \cdot I^{n}}{1+b_{1} \cdot I+b_{2} \cdot I^{2}+\cdots+b_{n} \cdot I^{n}} \cdot I .
\end{gathered}
$$

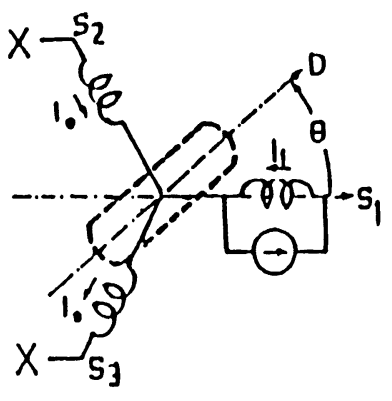

(a)

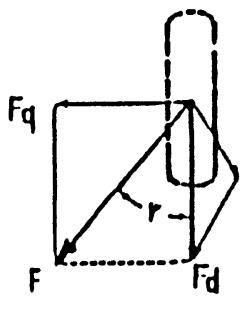

(b)

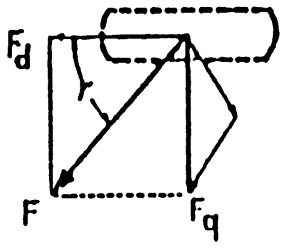

(c)

Fig. 1 bis. - Dispositif de mesure des inductances.

[Inductances measurements.] 
En ce qui concerne l'axe q, en plus des représentations analytiques de la forme (16), (17) et (20) une représentation logarithmique :

$$
\lambda(I)=a_{1} \log \left(\left(I-a^{2}\right) / b_{1}\right)
$$

peut être introduite.

D'autre part la courbe qui correspondrait correctement aux courbes expérimentales d'aimantation doit contenir trois parties distinctes :

- une partie linéaire pour des courants de faibles valeurs caractérisant l'inductance constante de nonsaturation,

- une partie non-linéaire pour des courants moyens,

- une autre partie linéaire pour des courants de fortes valeurs.

Afin d'approximer les courbes d'aimantation sur les axes d et q la fonction analytique (20) a été choisie. Ce choix semble être tout à fait convenable, car pour des valeurs faibles du courant la fonction dégénère en une droite $\lambda(I)=\left(a_{0} / 1\right)$. I qui correspond à la partie initiale de la courbe d'aimantation, et pour des valeurs fortes de courant elle a l'allure d'une autre droite $\lambda(I)=\left(a_{n} / b_{n}\right)$. I qui correspondrait à la partie finale de cette courbe. En effet on peut considérer la fonction (20) comme une suite de droites : $\quad \lambda(I)=\left(a_{0} / 1\right) . I, \quad$ puis : $\quad \lambda(I)=$ $\left(a_{1} / b_{1}\right)$. I puis $\lambda(I)=\left(a_{2} / b_{2}\right)$. I ... etc. avec une douce transition d'une droite à l'autre.

En gardant les deux droites, initiale et finale, on peut aussi approximer la partie non-linéaire de la courbe d'aimantation par une fonction exponentielle, hyperbolique ou logarithmique suivant l'axe $d$ ou q. Dans ce cas ces trois parties doivent être calculées de façon qu'aux points de jonction il n'y ait pas de discontinuité pour l'ensemble de la courbe ainsi que pour la première dérivée par rapport au courant. Afin de déterminer les paramètres de la fonction (20) le critère des moindres carrés a été retenu. Etant donné que la courbe expérimentale $\lambda_{\mathrm{d}}\left(I_{\mathrm{md}}\right)$ est déterminée pour $N$ paires de valeurs $\left(I_{\mathrm{md}}\right)_{i}$ et $\left(\lambda_{\mathrm{d}}\right)_{i}$, on calculera les paramètres de la fonction (20) $a_{0}, \ldots, a_{n}$ et $b_{1}, \ldots, b_{n}$ de façon à ce que cette fonction passe le plus près possible de ces $N$ points expérimentaux.

On calcule la distance de la fonction à chaque point c'est-à-dire l'erreur commise en chaque point et on détermine les coefficients de la fonction en minimisant l'erreur relative quadratique :

$$
\varepsilon(p)=\sum_{i=1}^{n}\left(\frac{\lambda_{\mathrm{d}_{i}}-\lambda_{\mathrm{d}_{i}}^{\prime}}{\lambda_{\mathrm{d}_{i}}^{\prime}}\right)^{2}
$$

dans laquelle $\lambda_{d}^{\prime}$ sont les valeurs expérimentales et $\lambda_{\mathrm{d}}$ sont les valeurs calculées.

Les conditions pour que $\varepsilon$ soit minimum sont alors :

$$
\begin{aligned}
& \frac{\partial \varepsilon}{\partial a_{0}}=0, \frac{\partial \varepsilon}{\partial a_{1}}=0, \ldots, \frac{\partial \varepsilon}{\partial a_{n}}=0, \frac{\partial \varepsilon}{\partial b_{1}}=0, \\
& \frac{\partial \varepsilon}{\partial b_{2}}=0, \ldots, \frac{\partial \varepsilon}{\partial b_{n}}=0
\end{aligned}
$$

soit :

$$
\nabla \varepsilon(p)=0
$$

Avec :

$$
P=\left(a_{0}, a_{1}, \ldots, a_{n}, b_{1}, b_{2}, \ldots, b_{n}\right)^{t}
$$

$P$ étant le vecteur des paramètres.

En substituant $\lambda_{\mathrm{d}_{i}}$ de l'équation (20), l'équation (22) devient :

$$
\begin{aligned}
& \varepsilon=\sum_{i=1}^{n}\left(\frac{1}{\lambda_{d_{i}}^{\prime 2}} \times\right. \\
& \left.\times\left(\frac{a_{0}+a_{1} I_{\mathrm{md}_{i}}+\cdots+a_{n} I_{\mathrm{md}_{i}}^{n}}{1+b_{1} I_{\mathrm{md}_{i}}+\cdots+b_{n} I_{\mathrm{md}_{i}}^{n}} I_{\mathrm{md}_{i}}-\lambda_{\mathrm{d}_{i}}^{\prime}\right)^{2}\right) \\
& =\sum_{i=1}^{n}\left(\begin{array}{c}
\left(a_{0} I_{\mathrm{md}_{i}}+a_{1} I_{\mathrm{md}_{i}}^{2}+\cdots+a_{n} I_{\mathrm{md}_{i}}^{n+1}\right)- \\
-\left(\lambda_{\mathrm{d}_{i}}^{\prime}+b_{1} \lambda_{\mathrm{d}_{i}}^{\prime} I_{\mathrm{md}_{i}}+\cdots+b_{n} \lambda_{\mathrm{d}_{i}}^{\prime} I_{\mathrm{md}_{i}}^{n}\right) \\
\lambda_{\mathrm{d}_{i}}^{\prime}\left(1+b_{1} I_{\mathrm{md}_{i}}+\cdots+b_{n} I_{\mathrm{md}_{i}}^{n}\right)
\end{array}\right)^{2} .
\end{aligned}
$$

Il faut résoudre $(2 n-1)$ équations pour $(2 n-1)$ paramètres $a_{0}, a_{1}, \ldots, a_{n}, b_{1}, b_{2}, \ldots, b_{n}$. Toutefois, les équations obtenues ne sont pas linéaires et pour les résoudre, il faut les linéariser. Ce système d'équations peut être linéarisé, si on remplace le dénominateur de (25) par une constante $P_{i}$ :

$$
P_{i}=\lambda_{\mathrm{d}_{i}}\left(1+b_{1} I_{\mathrm{md}_{i}}+b_{2} I_{\mathrm{md}_{i}}^{2}+\cdots+b_{n} I_{\mathrm{md}_{i}}^{n}\right)
$$

qui, initialement peut prendre la valeur 1 .

La fonction d'erreur devient donc :

$$
\begin{gathered}
\varepsilon=\sum_{i=1}^{n} \frac{1}{P_{i}^{2}}\left(\left(a_{0} I_{\mathrm{md}_{i}}+a_{1} I_{\mathrm{md}_{i}}^{2}+\cdots+a_{n} I_{\mathrm{md}_{i}}^{n+1}\right)-\right. \\
\left.-\left(1+b_{1} I_{\mathrm{md}_{i}}+\cdots+b_{n} I_{\mathrm{md}_{i}}^{n}\right) \lambda_{\mathrm{d}_{i}}^{\prime}\right)^{2}
\end{gathered}
$$

Avec $P_{i}$ constant, cette équation peut être facilement dérivée par rapport aux $(2 n-1)$ paramètres et on trouve $(2 n-1)$ équations linéaires pour déterminer les paramètres cherchés.

La courbe passe plus près des points expérimentaux lorsque la «densité » des points est plus grande. On peut commencer les calculs en donnant la valeur 1 aux constantes $P_{i}$ et par un calcul itératif, corriger sa valeur jusqu'à arriver à la convergence de l'itération.

On peut résoudre l'équation non linéaire (22) directement en utilisant la méthode de Marquardt. Cette méthode qui converge avec tout vecteur initial, est conseillée pour chercher le minimum des fonctions quadratiques. 
Afin de minimiser la fonction (22) il faut alors appliquer la formule suivante :

$$
p^{(k+1)}=p^{(k)}-\left(\nabla^{2} \varepsilon\left(p^{(k)}\right)+h_{11}^{(k)}\right) \nabla \varepsilon\left(p^{(k)}\right)
$$

ou $\nabla^{2} \varepsilon(p)$ est le Hessien de $\varepsilon(p), h$ est le pas automatique de calcul, 1 est la matrice unité d'ordre $(2 n-1)$ et $\nabla \varepsilon$ est le gradient de $\varepsilon(p)$.

$$
\begin{aligned}
& \nabla^{2} \varepsilon(p)=
\end{aligned}
$$

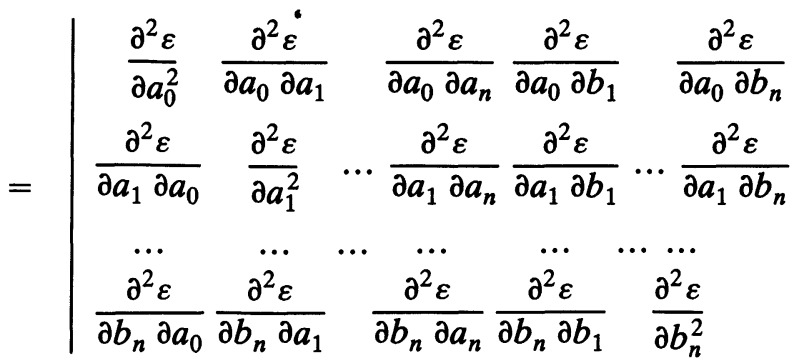

$$
\nabla \varepsilon(p)=\left|\frac{\partial \varepsilon}{\partial a_{0}}, \frac{\partial \varepsilon}{\partial a_{1}}, \ldots, \frac{\partial \varepsilon}{\partial a_{n}}, \frac{\partial \varepsilon}{\partial b_{1}}, \ldots, \frac{\partial \varepsilon}{\partial b_{n}}\right|^{t}
$$

En introduisant un vecteur $\boldsymbol{P}$ de valeurs arbitraires, l'équation (27) par itérations donnera de nouvelles valeurs de $P$, qui minimisent de mieux en mieux la fonction d'erreur (22) jusqu'à ce qu'on obtienne une erreur quadratique inférieure à une valeur initialement imposée.

Exemple d'application. - Les courbes expérimentales de l'aimantation magnétique sur l'axe $d$ et $q$ d'une machine synchrone à réluctance $2.5 \mathrm{~kW}$, $2 P=4$ pôles, $U_{\mathrm{n}}=220 \mathrm{~V}$ et $N=1000 \mathrm{tr} / \mathrm{mn}$ ont été relevées et sont présentées sur la figure 2 . Sur les mêmes figures, les courbes analytiques d'approximation avec différentes fonctions ont été représentées. La figure 3 montre la courbe expérimentale et la
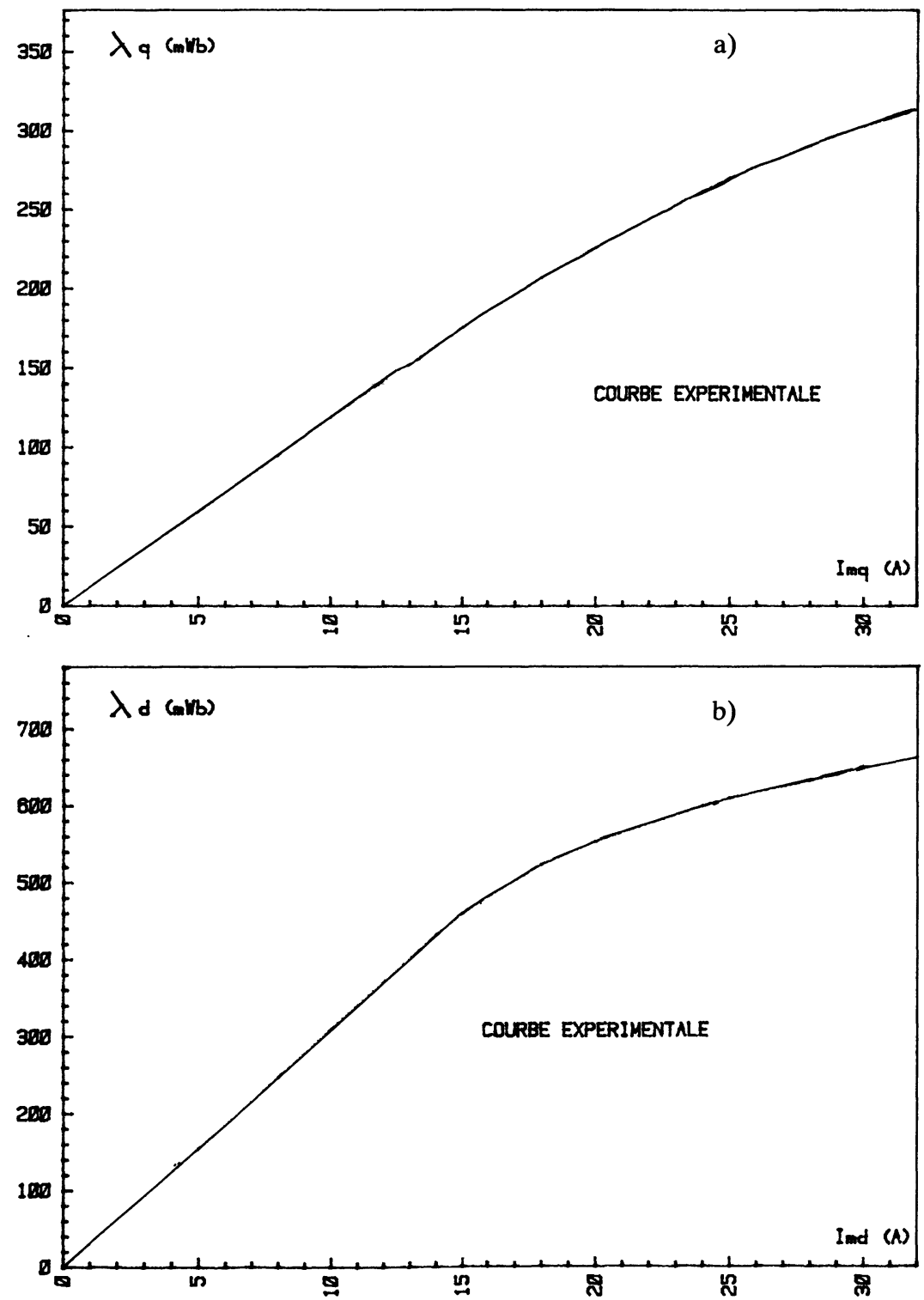

Fig. 2. - Les courbes expérimentales d'aimantation sur l'axe d (Fig. 2a) et q (Fig. 2b).

[Experimental magnetisation curves for $d$ and $q$-axes.] 


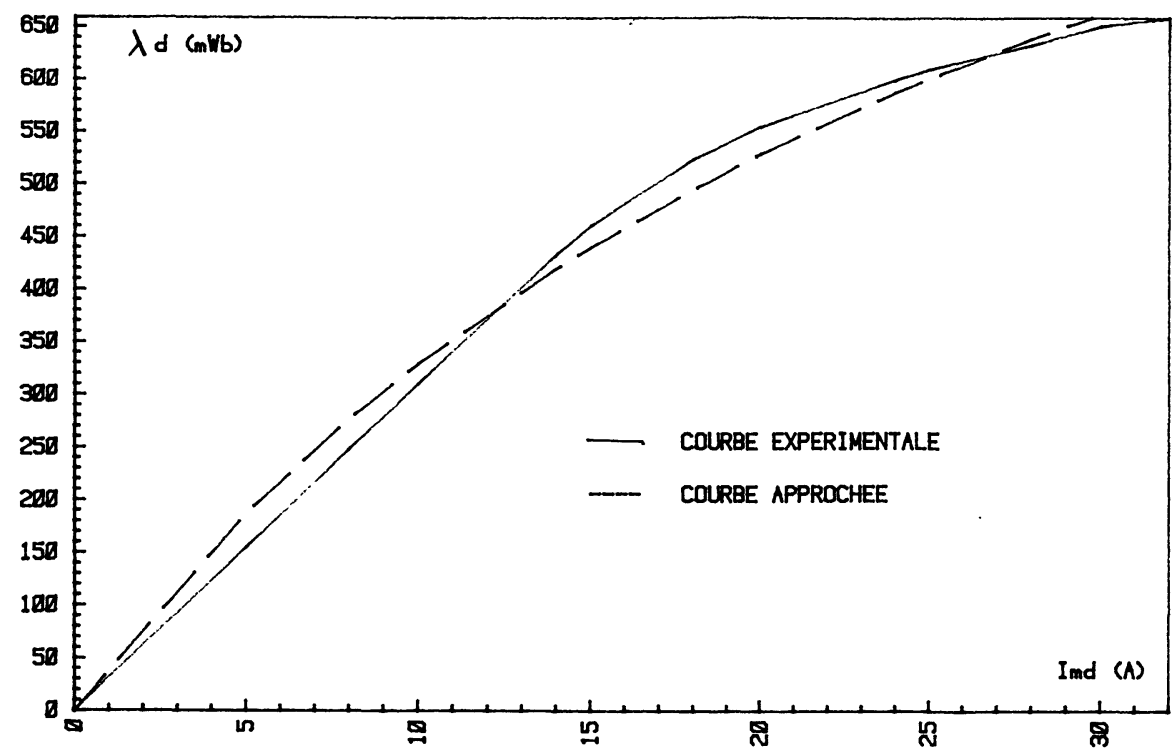

Fig. 3. - Représentation analytique de forme hyperbolique (16) de la courbe d'aimantation sur l'axe d. [Analytical representation of d-axis magnetisation curve by hyperbolic form of equation (16).]

courbe analytique hyperbolique de forme (20) alors que les figures 4 et 5 présentent la même courbe avec les courbes analytiques exponentielles de forme (17) et (18). Dans les figures 6 et 7 les mêmes comparaisons ont été faites pour les fonctions analytiques de forme (19) et (20) avec limitation des termes d'ordre 2. Les valeurs de paramètres qui étaient calculées par les méthodes ci-dessus mentionnées sont données ci-dessous :

pour la forme (19): $a_{1}=22,269$

$$
\begin{aligned}
& a_{2}=1,6201 \\
& b_{1}=0 \\
& b_{2}=2,509 \times 10^{-3}
\end{aligned}
$$

pour la forme (20): $a_{0}=35,509$

$$
\begin{aligned}
& a_{1}=-0,6732 \\
& b_{1}=-9,795 \times 10^{-3} .
\end{aligned}
$$

On a observé que la simplicité de la fonction approximant la courbe expérimentale ne coïncide pas toujours avec la meilleure qualité d'approximation. D'autre part il faut considérer le fait que les courbes, bien qu'elles soient convenables pour la simulation des machines aux alentours des points de fonctionnement de ces machines donnent des résultats erronés au-delà de la valeur nominale du courant. Donc, afin de trouver une fonction qui réponde aux besoins de modélisation des machines

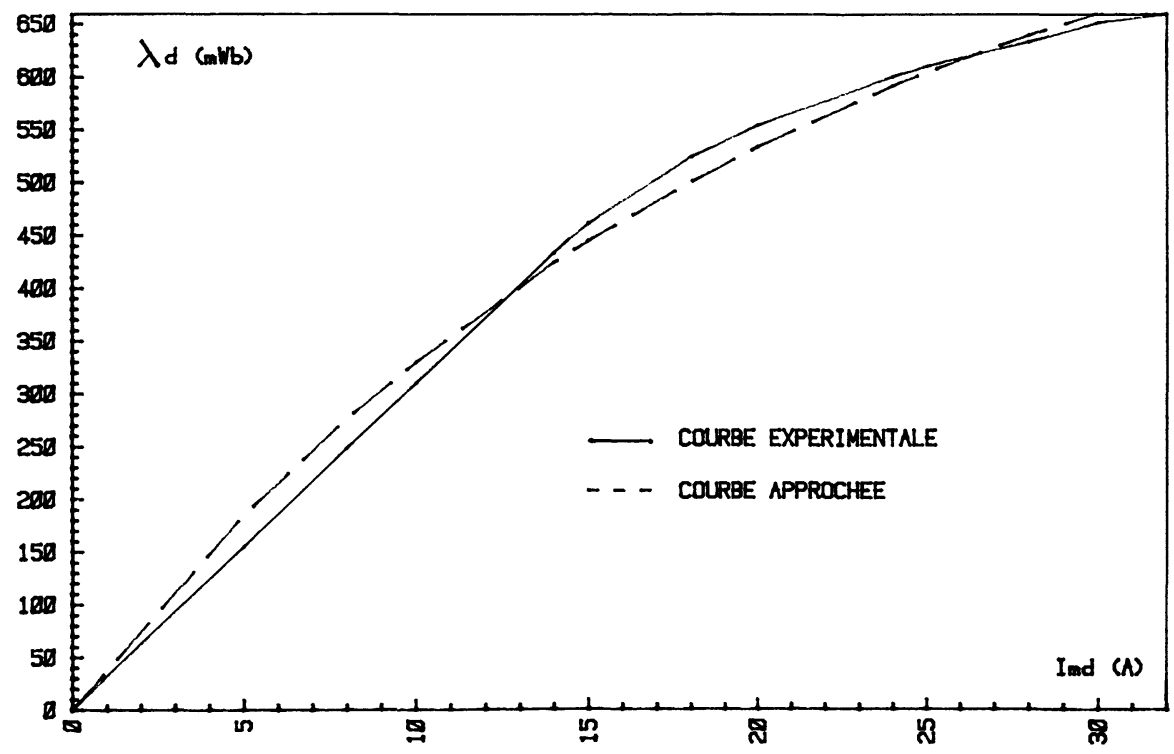

Fig. 4. - Représentation analytique de forme exponentielle (17) de la courbe d'aimantation sur l'axe d. [Analytical representation of d-axis magnetisation curve by exponential form of equation (17).] 


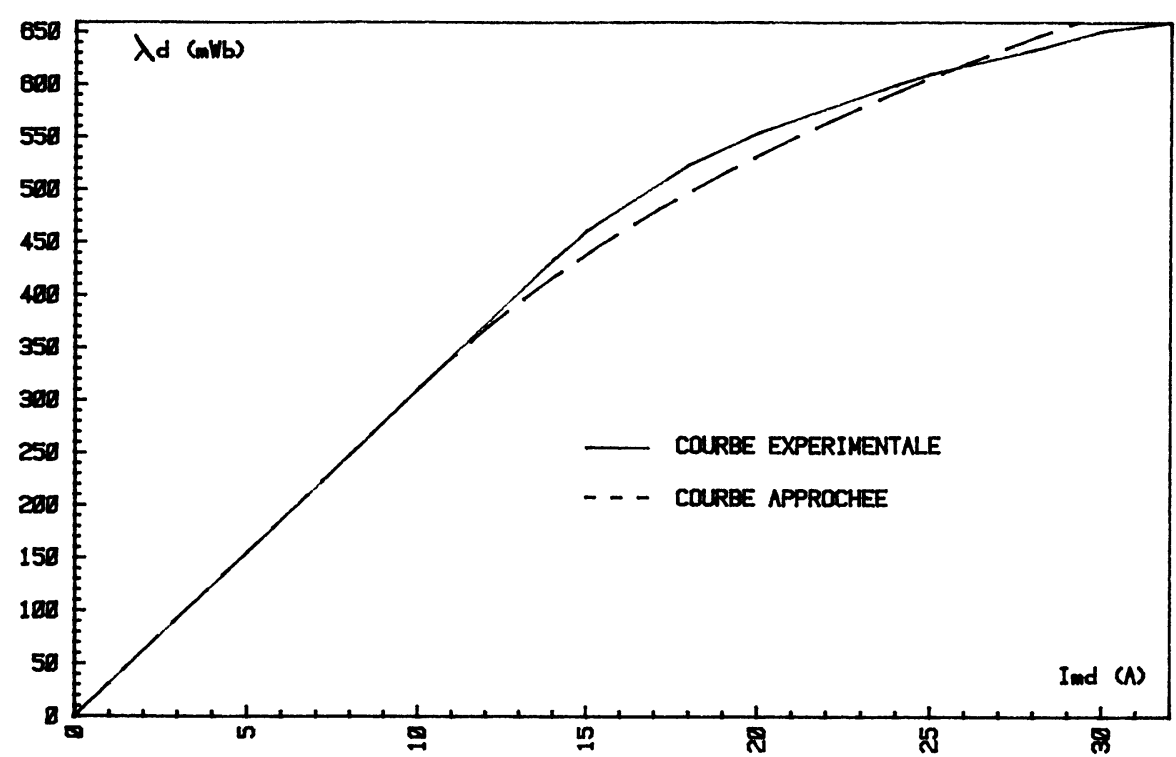

Fig. 5. - Représentation analytique de forme exponentielle (18) de la courbe d'aimantation sur l'axe d. [Analytical representation of d-axis magnetisation curve by exponential form of equation (18).]

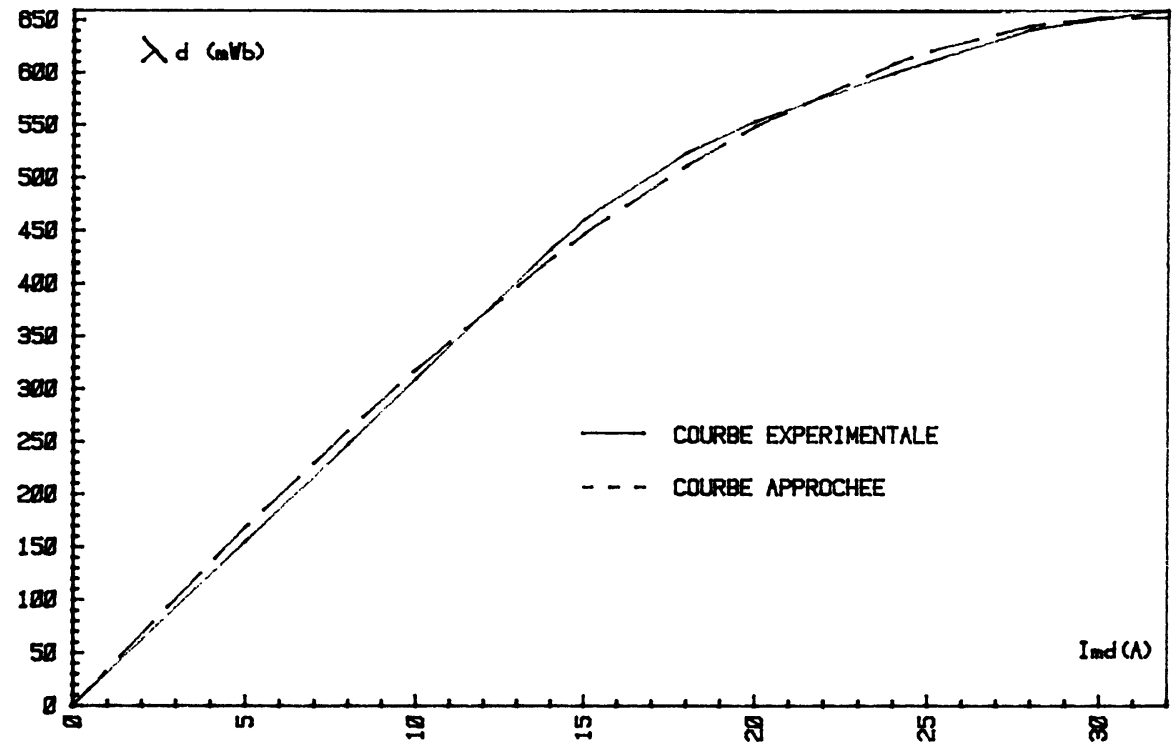

Fig. 6. - Représentation analytique de forme fraction polynomiale (19) de la courbe d'aimantation sur l'axe d. [Analytical representation of d-axis magnetisation curve by rational-fraction form of equation (19).]

dans une gamme très large de courant et qui puisse être utilisée aussi bien pour l'étude des régimes permanents que pour des régimes transitoires, il faut ajouter quelques points dans la partie supérieure de la courbe et extrapoler la courbe linéairement pour donner une gamme plus large pour les courants.

D'où l'idée, à partir d'une valeur donnée de courant (généralement supérieure à la valeur nominale), de continuer avec une droite dont la pente coïnciderait avec celle de la courbe réelle lorsque le courant tend vers des valeurs très grandes. On a observé aussi, que pour toutes les fonctions choisies, pour des valeurs faibles de courant on n'observe pas une partie vraiment linéaire. On va donc essayer de construire la courbe en trois parties, deux parties linéaires et au milieu une partie non linéaire. Or, ces trois parties doivent être calculées de façon qu'aux points de jonctions il n'y ait pas de discontinuité pour la courbe elle-même, ainsi que pour la première dérivée par rapport au courant. Cela amène la continuité des valeurs des inductances statiques et dynamiques et par conséquent évite les erreurs dues aux discontinuités de ces dernières dans les simulations. 


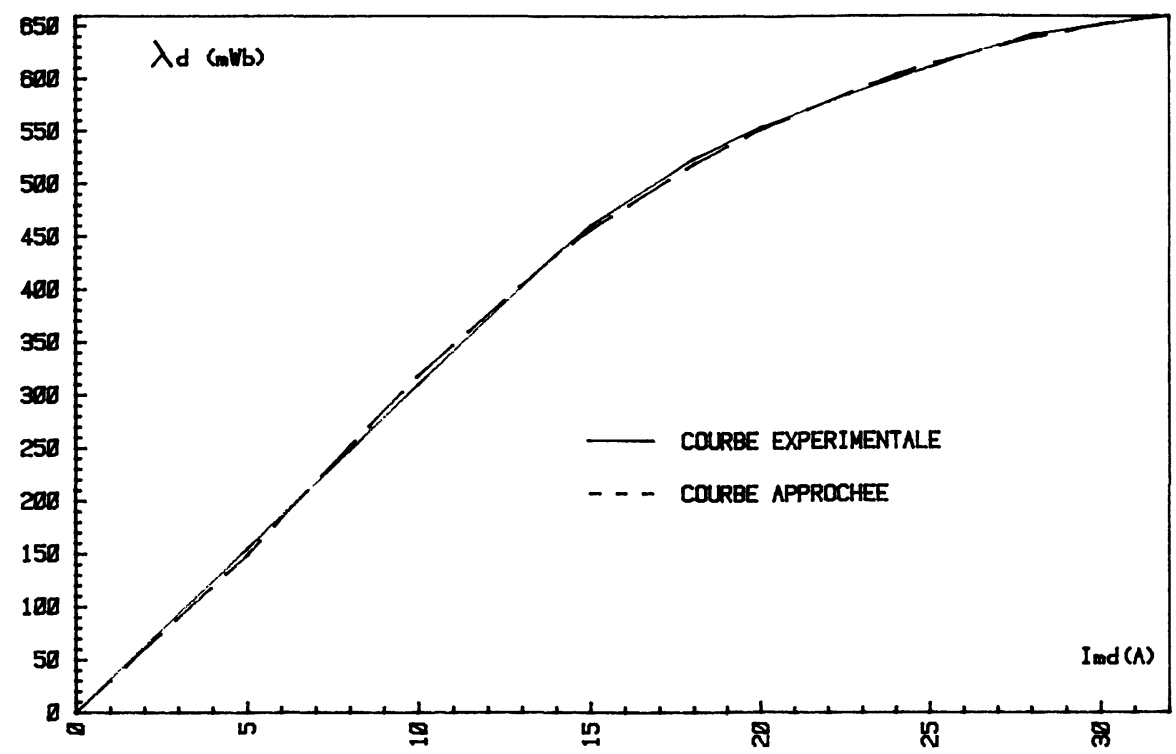

Fig. 7. - Représentation analytique de forme fraction-polynomiale (20) de la courbe d'aimantation sur l'axe d.

[Analytical representation of d-axis magnetisation curve by rational-fraction form of equation (20).]

La figure 8 montre la courbe expérimentale et la courbe analytique « trois parties », avec un minimum d'écart entre elles.

En présentant la courbe de saturation sous la forme de l'équation (19) :

$$
\lambda_{\mathrm{md}}=\frac{a_{1} I_{\mathrm{md}}+a_{2} I_{\mathrm{md}}^{2}}{1+b_{2} I_{\mathrm{md}}^{2}}
$$

et en utilisant une méthode de minimisation conditionnée on peut mentionner les différentes contraintes. La première est :

$$
g_{1}=\operatorname{tg} \beta I_{\mathrm{md}_{1}}-\frac{a_{1} I_{\mathrm{md}_{1}}+a_{2} I_{\mathrm{md}_{1}}^{2}}{1+b_{2} I_{\mathrm{md}_{1}}^{2}}=0 .
$$

Cette contrainte garantit la continuité de la première partie linéaire et de la partie non linéaire de la courbe au point $I_{\mathrm{md}_{1}}$. Ce point étant la limite de la partie linéaire dans laquelle $\operatorname{tg} \beta$ est égale à l'inductance statique de la partie linéaire pour des valeurs faibles de courant.

La deuxième contrainte peut être écrite :

$$
g_{2}=\operatorname{tg} \beta-\frac{\partial \lambda_{\mathrm{md}}}{\partial I_{\mathrm{md}}}\left(\text { pour } I_{\mathrm{md}}=I_{\mathrm{md}_{1}}\right)=0 .
$$

Cette contrainte garantit la continuité de la dérivée par rapport au courant des deux premières parties de la courbe au point de jonction.

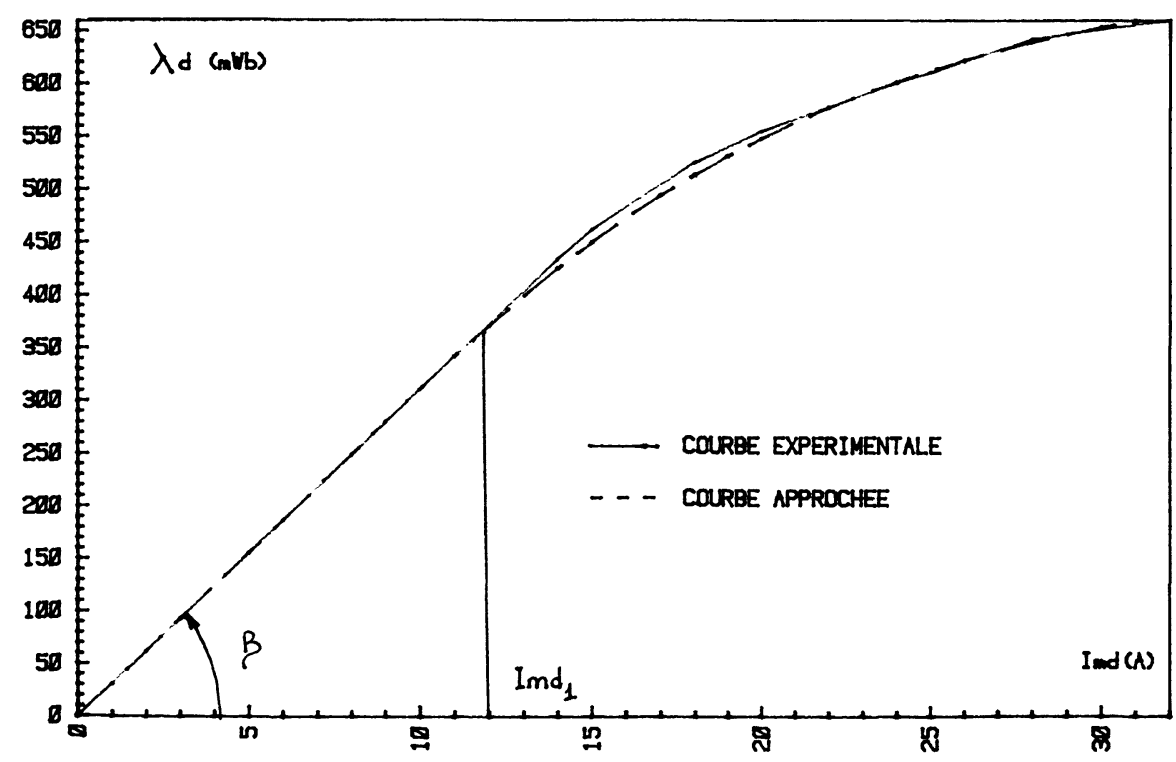

Fig. 8. - Représentation analytique trois parties de la courbe d'aimantation sur l'axe d.

[Analytical representation of d-axis magnetisation curve by the three parts form.] 
Considérant les deux contraintes et la fonction d'erreur relative quadratique, selon les multiplieurs de Lagrange, on peut écrire :

$$
f(p, \alpha)=\varepsilon(p)+\sum_{i=1}^{2} \alpha_{i} g_{i}
$$

En minimisant la fonction (28) on détermine le vecteur $P$ et par conséquent l'expression analytique de la deuxième partie non linéaire de la courbe analytique.

Pour tracer la troisième partie de cette courbe, on calcule $I_{\text {md }_{\max }}$ tel que :

$$
\frac{\partial \lambda_{\mathrm{md}}}{\partial I_{\mathrm{md}}}\left(\text { pour } I_{\mathrm{md}}=I_{\mathrm{md}_{\max }}\right)=0 .
$$

Au-delà de $K_{\mathrm{d}} \cdot I_{\text {md }_{\max }}$ (avec $K_{\mathrm{d}}=0,9$ par exemple) on continue avec une droite dont la pente est égale à celle de la tendance de la courbe réelle lorsque $I_{\mathrm{md}}$ tend vers des valeurs très importantes.

Les figures 9, 10, 11 correspondent aux courbes expérimentales et analytiques sur l'axe q en utilisant les fonctions (16), (17), (20). Quant à la figure 12 elle montre la courbe d'aimantation sur l'axe q en comparaison avec la courbe analytique logarithmique de la forme (21).

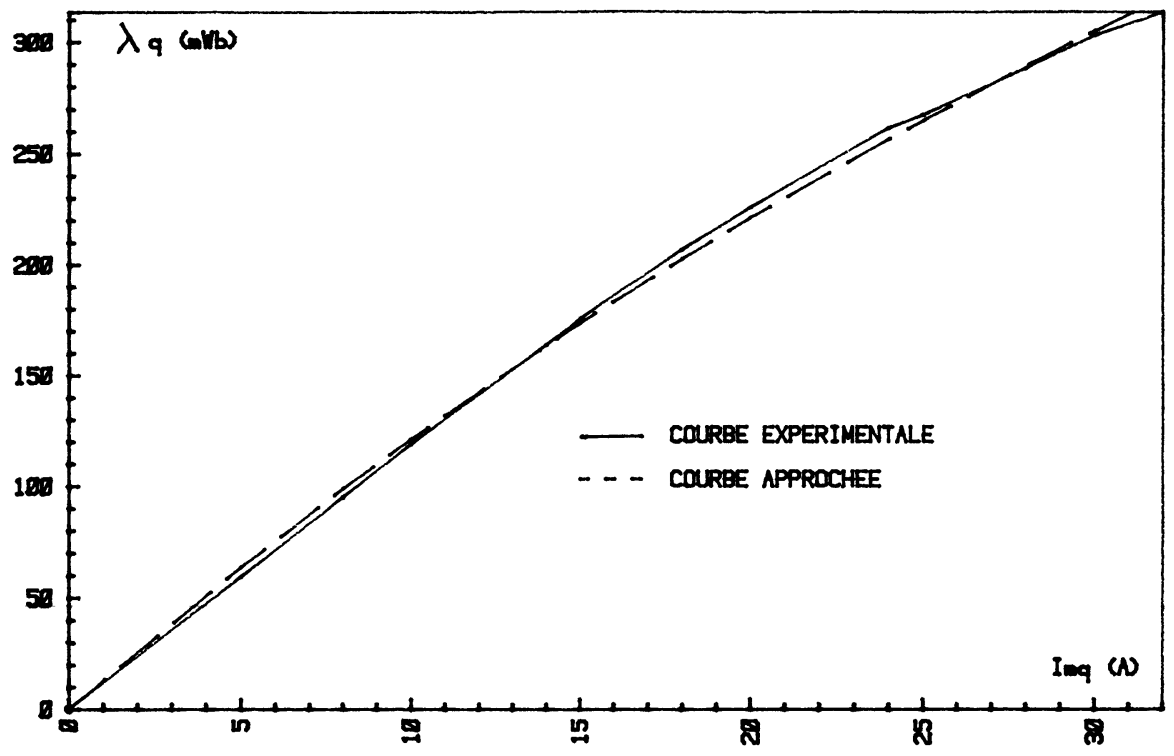

Fig. 9. - Représentation analytique de forme hyperbolique (16) de la courbe d'aimantation sur l'axe q. [Analytical representation of q-axis magnetisation curve by hyperbolic form of equation (16).]

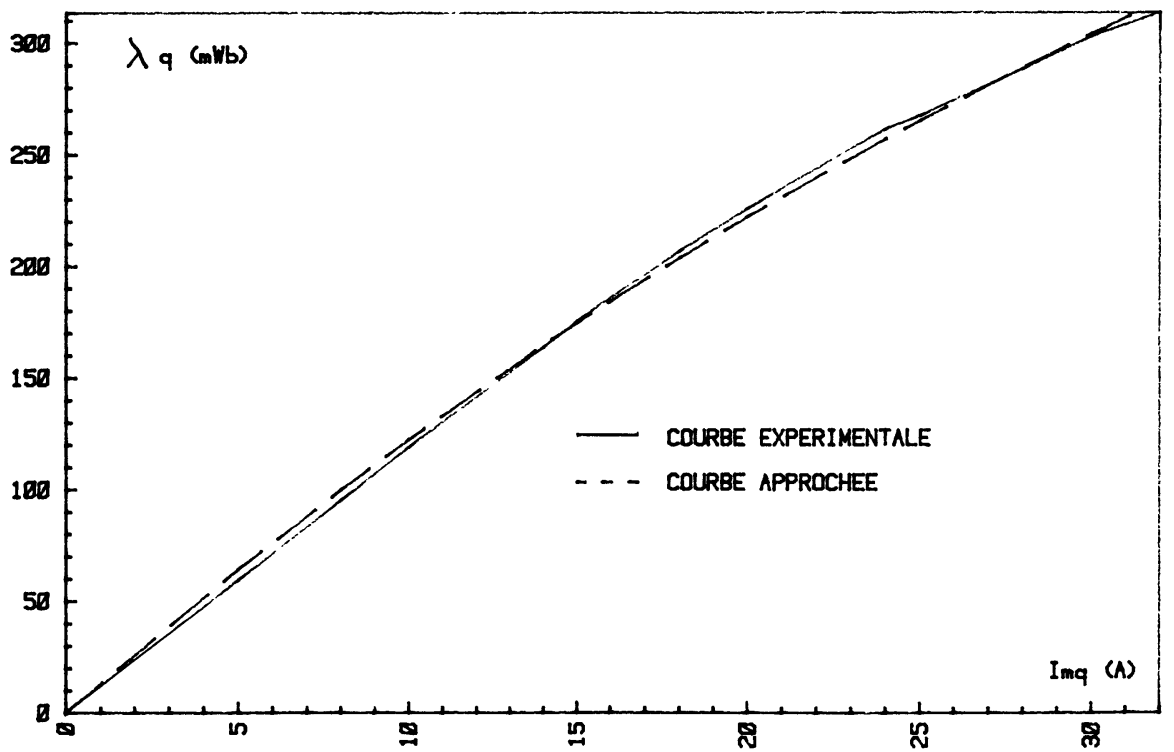

Fig. 10. - Représentation analytique de forme exponentielle (17) de la courbe d'aimantation sur l'axe q. [Analytical representation of $q$-axis magnetisation curve by exponential form of equation (17).] 


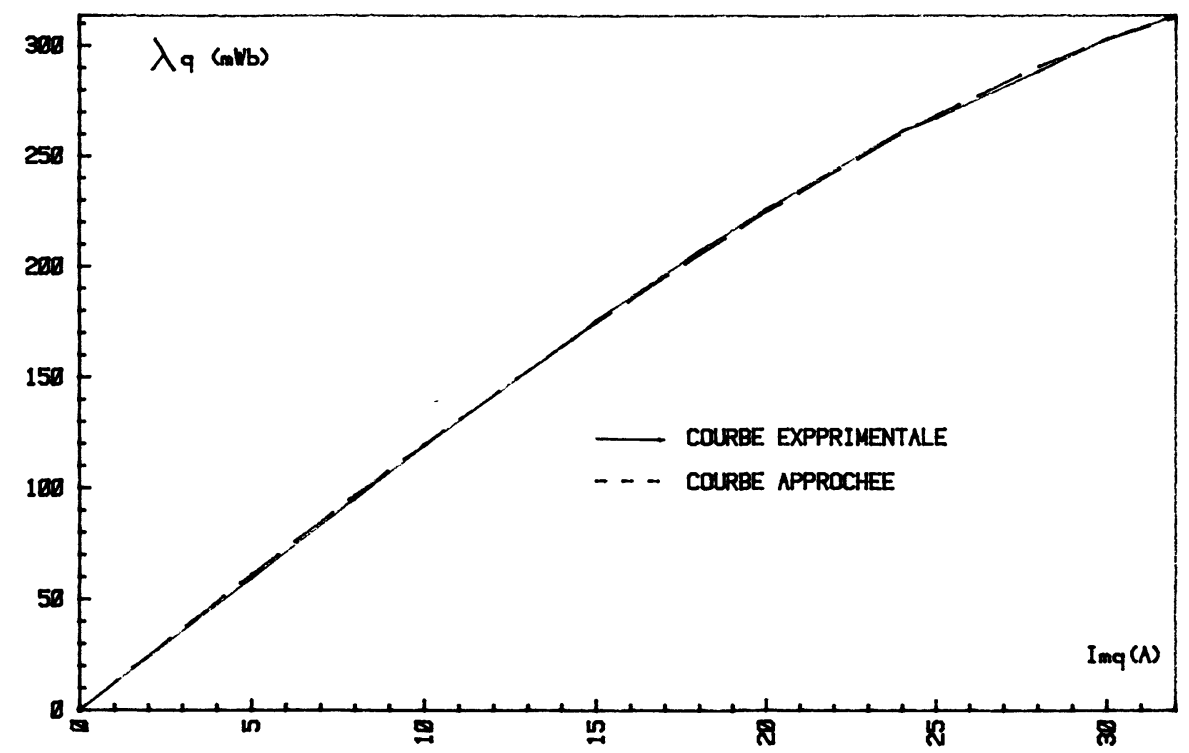

Fig. 11. - Représentation analytique de forme fraction-polynomiale (20) de la courbe d'aimantation sur l'axe q. [Analytical representation of q-axis magnetisation curve by rational-fraction form of equation (20).]

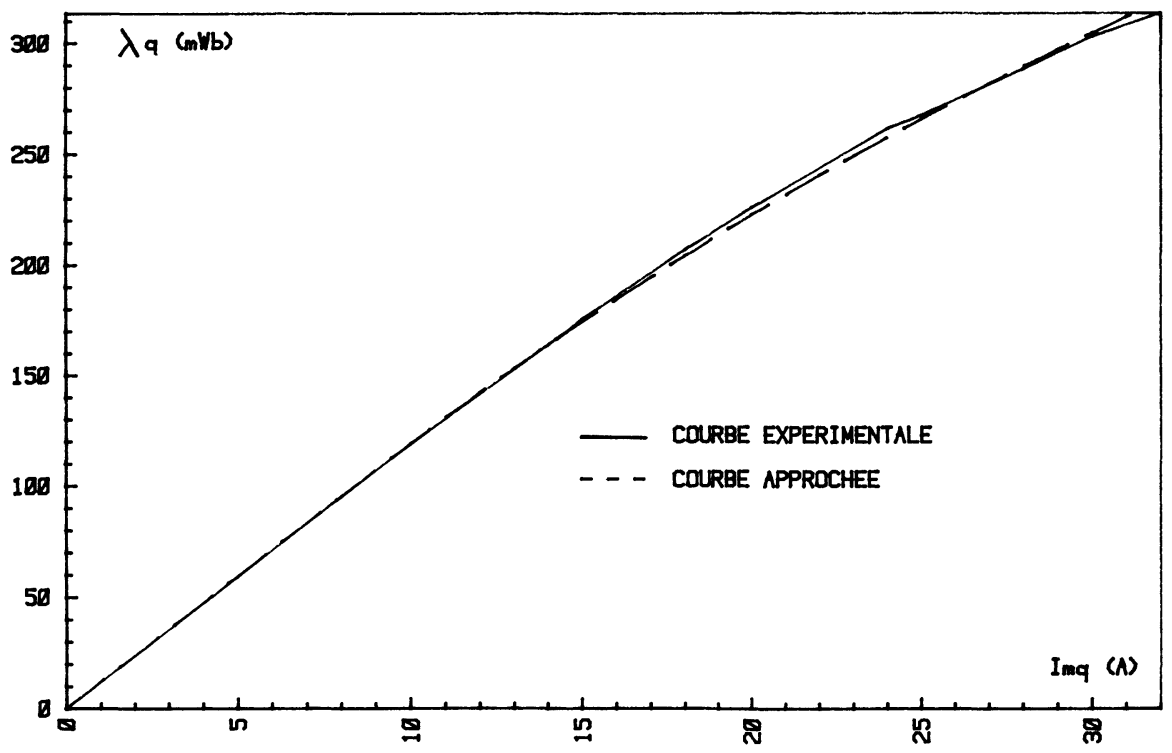

Fig. 12. - Représentation analytique de forme logarithmique (21) de la courbe d'aimantation sur l'axe q.

[Analytical representation of q-axis magnetisation curve by logarithmic form of equation (21).]

3.2 REPRÉSENTATION DES SURFACES D'AIMANTATION. - En considérant l'effet croisé de saturation, d'après les relations (5) et (6), on peut essayer de représenter analytiquement la surface $\lambda_{\mathrm{d}}=f$ $\left(I_{\mathrm{md}}, I_{\mathrm{mq}}\right)$ dans le système de coordonnées $I_{\mathrm{md}}$, $I_{\mathrm{mq}}, \lambda_{\mathrm{d}}$ et la surface $\lambda_{\mathrm{q}}$ dans le système de coordonnées $I_{\mathrm{mq}}, I_{\mathrm{md}}, \lambda_{\mathrm{q}}$ comme on le montre dans les figures 13 et 14 .

Afin de représenter analytiquement la surface $\lambda_{\mathrm{d}}=f\left(I_{\mathrm{md}}, I_{\mathrm{mq}}\right)$ on peut trouver les traces de cette surface dans les plans $I_{\mathrm{mq}}=$ cte. La trace de cette surface sur le plan $I_{\mathrm{mq}}=0$ est déjà calculée, elle est en fait la courbe d'aimantation de la machine sur l'axe d sans considérer l'effet croisé. Il nous suffit d'abord de trouver expérimentalement les courbes de saturation sur cet axe d pour différentes valeurs de courant sur l'axe $q$ et ensuite représenter ces courbes analytiquement comme on l'a déjà vu au chapitre précédent. On trouvera ainsi les traces de la surface d'aimantation sur les plans $I_{\mathrm{mq}}=$ cte. Pour éviter des calculs trop longs on répètera deux fois cette procédure pour deux valeurs extrêmes de $I_{\mathrm{mq}}$ pour lesquelles on dispose des données expérimentales, l'une correspond à $I_{\mathrm{mq}}=0$ et l'autre à $I_{\mathrm{mq}}=I_{\mathrm{n}}$ dans laquelle $I_{\mathrm{n}}$ est le courant nominal de la machine. Pour des valeurs intermédiaires de $I_{\mathrm{mq}}$ et 


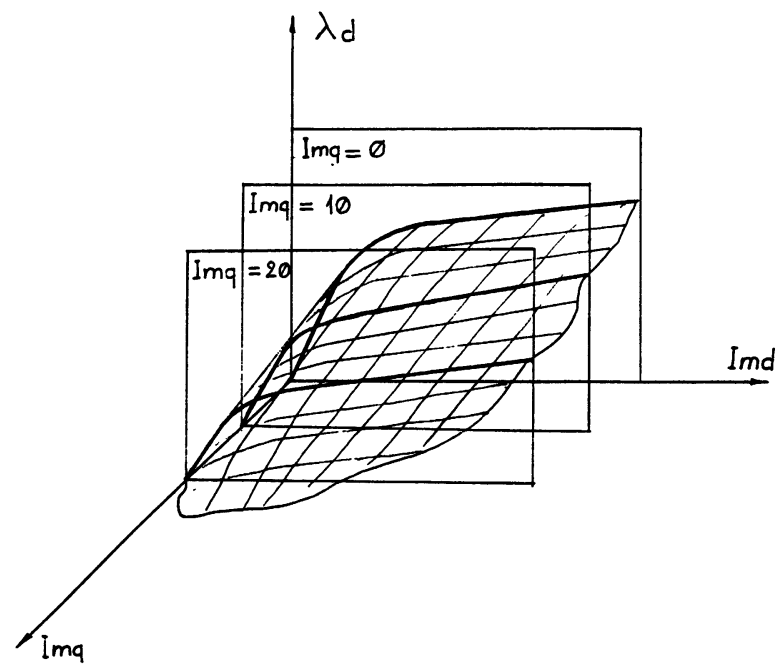

Fig. 13. - Surface d'aimantation $\lambda_{\mathrm{d}}=f\left(I_{\mathrm{md}}, I_{\mathrm{mq}}\right)$.

[Magnetisation surface $\lambda_{\mathrm{d}}=f\left(I_{\mathrm{md}}, I_{\mathrm{mq}}\right)$.]

par conséquent pour trouver les traces dans les plans intermédiaires on procède de la manière suivante : dans les formules de (16) à (20) au lieu de considérer les paramètres $a_{0}, a_{1}, \ldots, a_{n}, b_{1}, \ldots, b_{n}$ comme constantes, on peut les présenter sous forme d'une fonction du courant $I_{\mathrm{mq}}$ :

$$
\begin{aligned}
& a_{\mathrm{d}_{i}}=a_{\mathrm{d}_{i 0}}+a_{d_{i 1}} I_{\mathrm{mq}}^{2} \\
& b_{\mathrm{d}_{i}}=b_{\mathrm{d} 0}+b_{\mathrm{d}_{i 1}} I_{\mathrm{mq}}^{2} .
\end{aligned}
$$

Ainsi les formules de (16) à (20) ne représentent pas une courbe mais une surface et pour trouver les traces de celle-ci dans les plans $I_{\mathrm{mq}}=$ cte il suffit de mettre la valeur correspondante de $I_{\mathrm{mq}}$ dans les équations modifiées de (16) à (20).

La même procédure peut être appliquée pour présenter la surface $\lambda_{\mathrm{q}}=f\left(I_{\mathrm{mq}}, I_{\mathrm{md}}\right)$, en supposant que les paramètres de la fonction (18) et (19) sont :

$$
\begin{aligned}
& a_{\mathrm{q}_{i}}=a_{\mathrm{q}_{\mathrm{i}}}+a_{\mathrm{q}_{i 1}} I_{\mathrm{md}}^{2} \\
& b_{\mathrm{q}_{i}}=b_{\mathrm{q}_{\mathrm{i} 0}}+b_{\mathrm{q}_{\mathrm{i} 1}} I_{\mathrm{md}}^{2} .
\end{aligned}
$$

En choisissant la fonction (20) pour représenter les surfaces $\lambda_{\mathrm{d}}$ et $\lambda_{\mathrm{q}}$ les expressions analytiques deviennent alors :

$$
\begin{gathered}
\lambda_{\mathrm{d}}\left(I_{\mathrm{md}}, I_{\mathrm{mq}}\right)=\frac{\left(a_{\mathrm{d}_{00}}+a_{\mathrm{d}_{01}} I_{\mathrm{mq}}^{2}\right)+\left(a_{\mathrm{d}_{10}}+a_{\mathrm{d}_{11}} I_{\mathrm{mq}}^{2}\right) I_{\mathrm{md}}+\cdots}{1+\left(b_{\mathrm{d}_{10}}+b_{\mathrm{d}_{11}} I_{\mathrm{mq}}^{2}\right) I_{\mathrm{md}}+\left(b_{\mathrm{d}_{20}}+b_{\mathrm{d}_{21}} I_{\mathrm{mq}}^{2}\right) I_{\mathrm{md}}^{2}+\cdots} I_{\mathrm{md}} \\
\lambda_{\mathrm{q}}\left(I_{\mathrm{md}}, I_{\mathrm{mq}}\right)=\frac{\left(a_{\mathrm{q}_{00}}+a_{\mathrm{q}_{01}} I_{\mathrm{md}}^{2}\right)+\left(a_{\mathrm{q}_{10}}+a_{\mathrm{q}_{11}} I_{\mathrm{md}}^{2}\right) I_{\mathrm{mq}}+\cdots}{1+\left(b_{\mathrm{q}_{10}}+b_{\mathrm{q}_{11}} I_{\mathrm{md}}^{2}\right) I_{\mathrm{mq}}+\left(b_{\mathrm{q}_{20}}+b_{\mathrm{q}_{21}} I_{\mathrm{mq}}^{2}\right) I_{\mathrm{mq}}^{2}+\cdots} I_{\mathrm{md}} .
\end{gathered}
$$

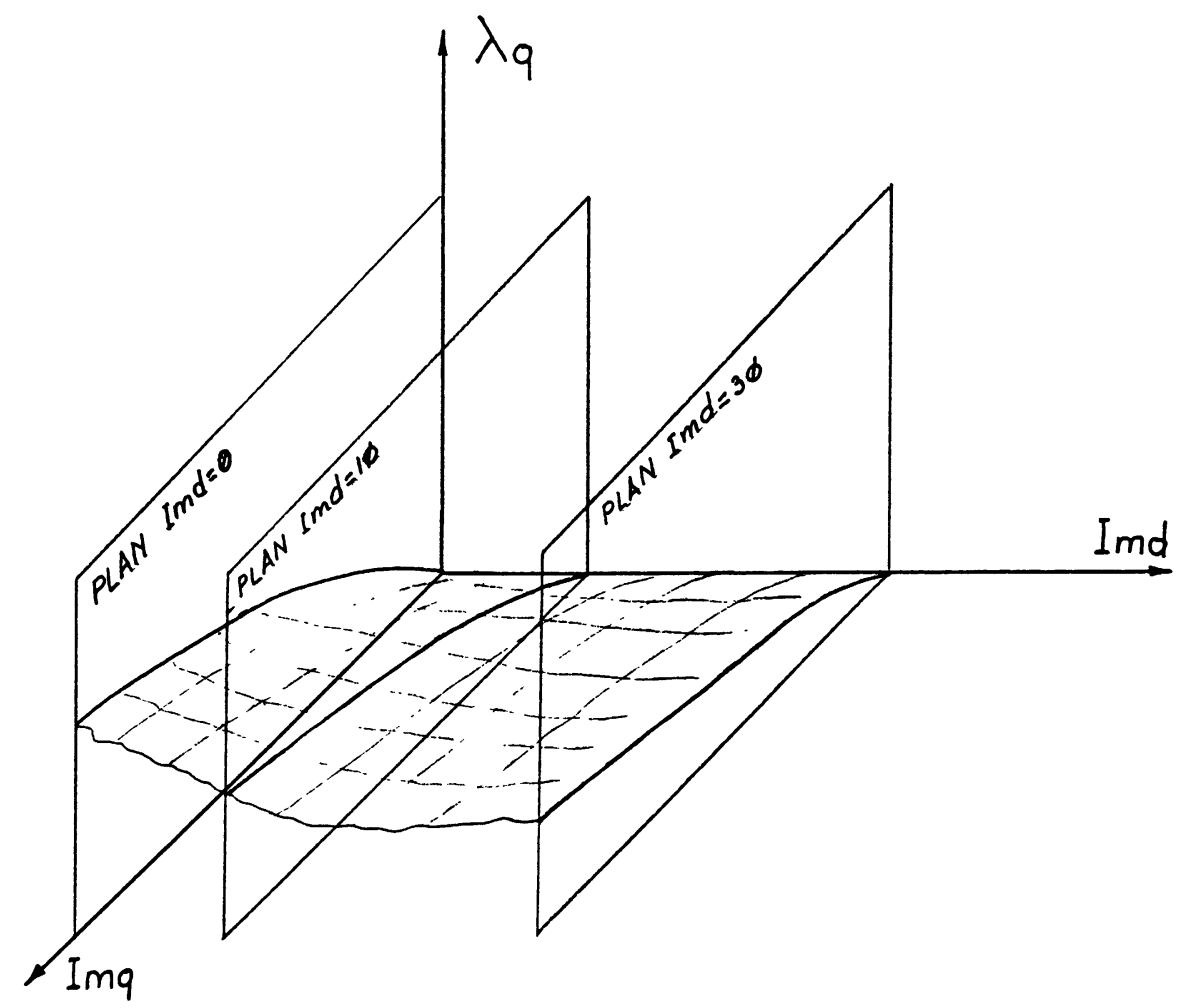

Fig. 14. - Surface d'aimantation $\lambda_{\mathrm{q}}=f\left(I_{\mathrm{mq}}, I_{\mathrm{md}}\right)$.

[Magnetisation surface $\lambda_{\mathrm{d}}=f\left(I_{\mathrm{mq}}, I_{\mathrm{md}}\right)$.] 


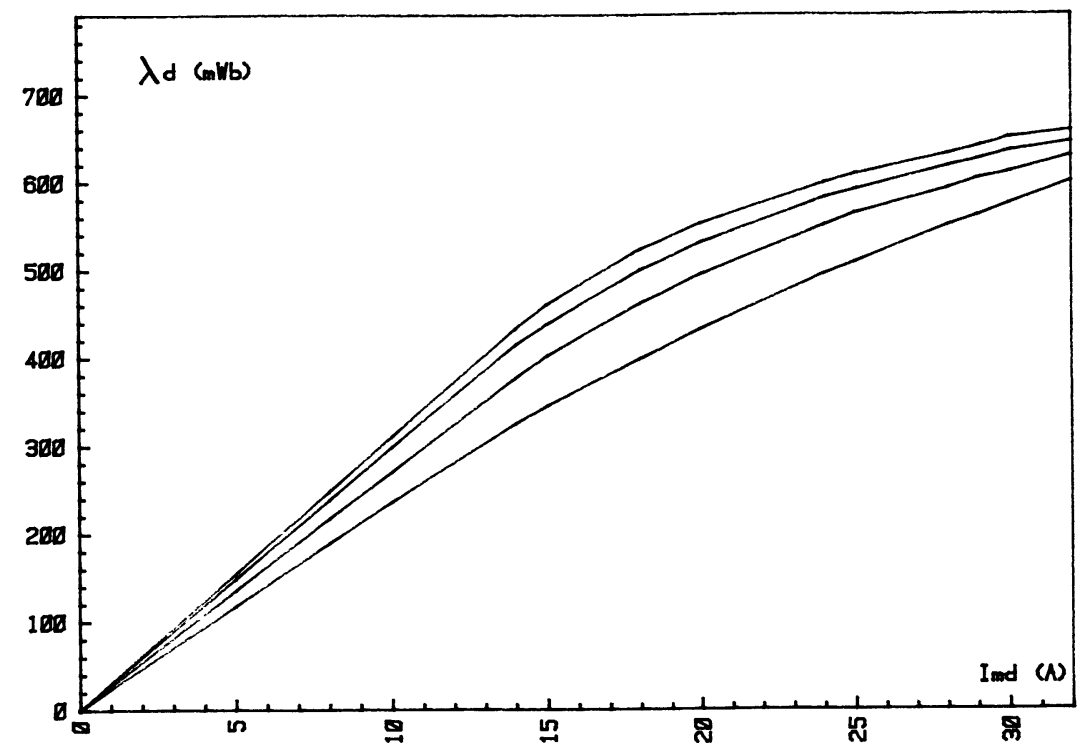

Fig. 15. - Courbes expérimentales d'aimantation $\lambda_{\mathrm{d}}\left(I_{\mathrm{md}}, I_{\mathrm{mq}}\right)$.

[The experimental magnetisation curves $\lambda_{\mathrm{d}}\left(I_{\mathrm{md}}, I_{\mathrm{mq}}\right)$.]

Afin de déterminer tous les coefficients, on applique la méthode de Marquardt (la formule (27)). Ainsi on peut calculer les valeurs de $a_{\mathrm{d}_{i}}, b_{\mathrm{d}_{i}}$ et $a_{\mathrm{q}_{i}}, b_{\mathrm{q}_{i}}$ et en considérant que $a_{\mathrm{d}_{0}}, b_{\mathrm{d}_{0}}, a_{\mathrm{q}_{0}}$ et $b_{\mathrm{q}_{0}}$ étaient déjà calculés et que les valeurs de $I_{\mathrm{md}}$ et $I_{\mathrm{mq}}$ sont connues, on peut en déduire à partir des équations (29) et (30) $a_{\mathrm{d}_{i 1}}, a_{\mathrm{q}_{11}}, b_{\mathrm{d}_{i 1}}$ et $b_{\mathrm{q}_{i 1}}$ $(i=0,1,2, \ldots, n)$.

La figure 16 montre les projections des traces de la surface d'aimantation $\lambda_{\mathrm{d}}$ dans des plans $I_{\mathrm{mq}}=$ cte sur le plan $I_{\mathrm{mq}}=0$. De la même manière la figure 18 montre la famille des courbes d'aimantation sur l'axe q.

Exemple d'application. - La représentation analytique de la surface d'aimantation sur les deux axes pour la machine à reluctance mentionnée au chapitre précédent avec le choix de la meilleure fonction approximant les données expérimentales est la suivante :

Pour l'axe $\mathrm{d}$ avec la représentation en trois parties et $\lambda_{d}$ en $\mathrm{mWb}$ :

- une partie linéaire $\lambda_{\mathrm{d}}=k_{1} \cdot I_{\mathrm{md}}$

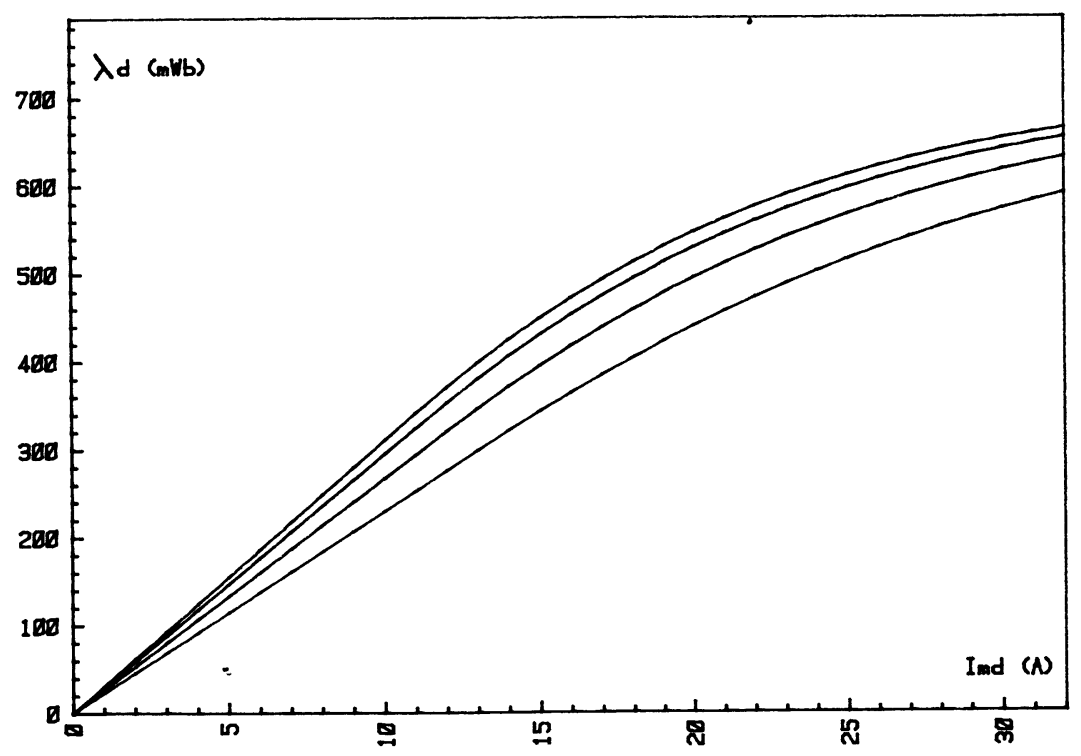

Fig. 16. - La projection des traces de la surface d'aimantation $\lambda_{\mathrm{d}}=f\left(I_{\mathrm{md}}, I_{\mathrm{mq}}\right)$ sur le plan $I_{\mathrm{mq}}=0$.

[The projection traces of the magnetisation surface $\lambda_{\mathrm{d}}=f\left(I_{\mathrm{md}}, I_{\mathrm{mq}}\right)$ over the plane $I_{\mathrm{mq}}=0$.] 


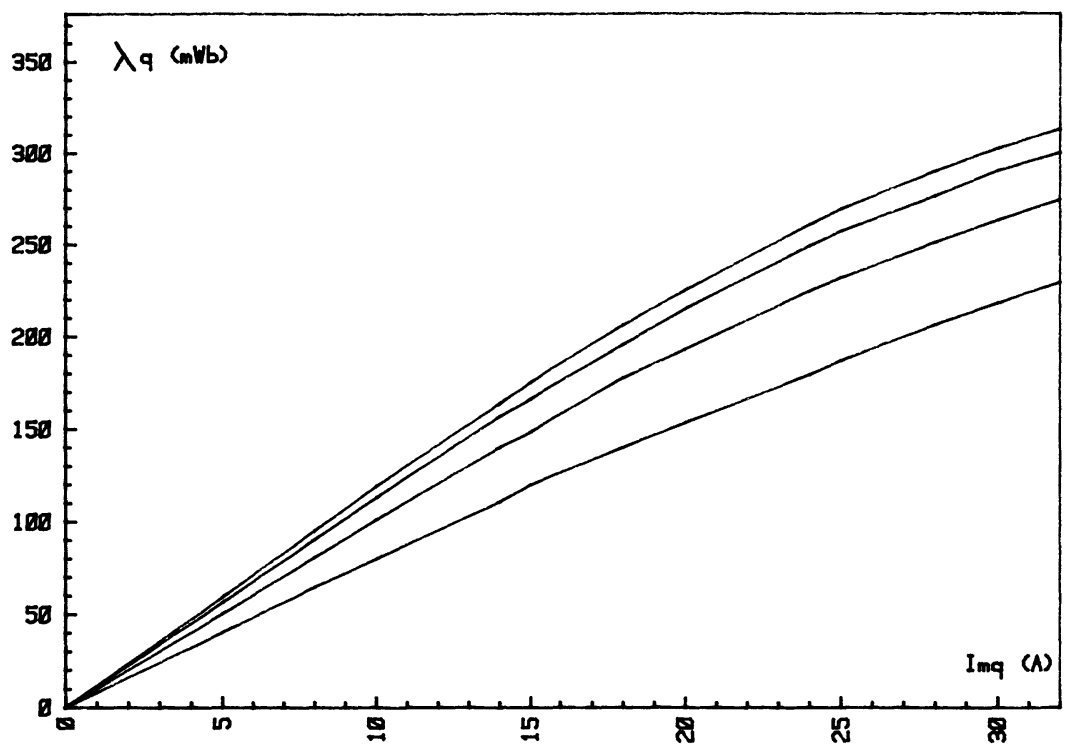

Fig. 17. - Courbes expérimentales d'aimantation $\lambda_{\mathrm{q}}\left(I_{\mathrm{md}}, I_{\mathrm{mq}}\right)$.

[The experimental magnetisation curves $\lambda_{\mathrm{q}}\left(I_{\mathrm{md}}, I_{\mathrm{mq}}\right)$.]

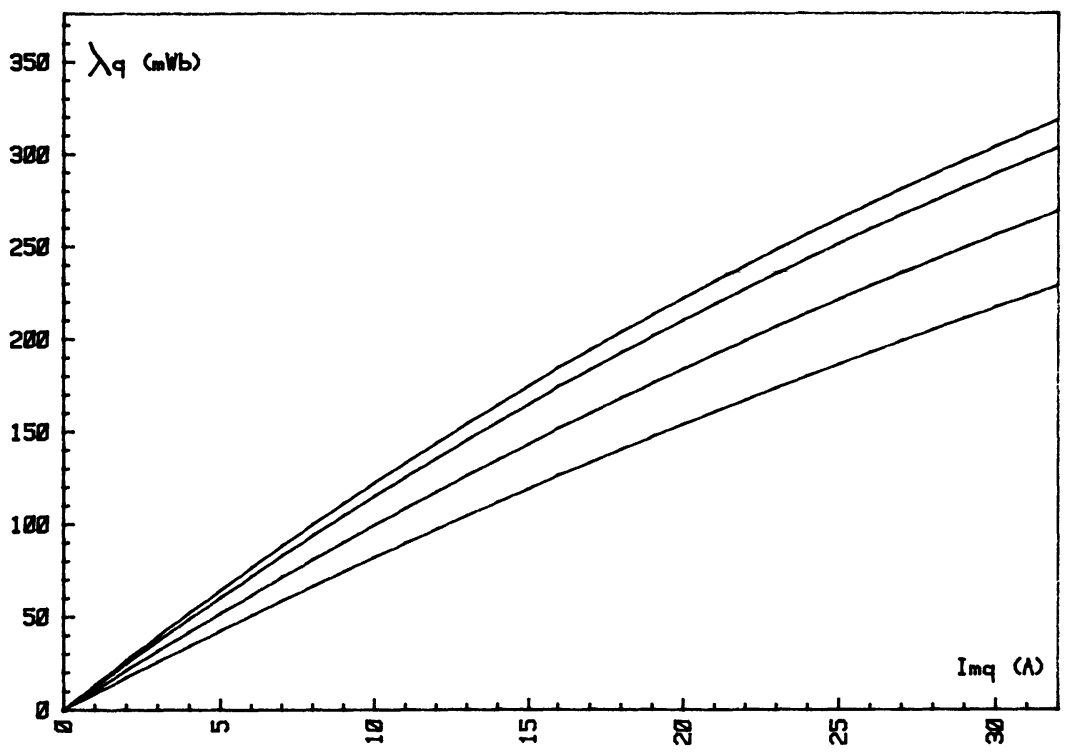

Fig. 18. - La projection des traces de la surface d'aimantation $\lambda_{\mathrm{q}}=f\left(I_{\mathrm{mq}}, I_{\mathrm{md}}\right)$ sur le plan $I_{\mathrm{md}}=0$.

[The projection traces of the magnetisation surface $\lambda_{\mathrm{q}}=f\left(I_{\mathrm{mq}}, I_{\mathrm{md}}\right)$ over the plane $I_{\mathrm{md}}=0$.]

- une partie non linéaire avec la fonction (19) limitée à l'ordre 2 :

$$
\begin{aligned}
\lambda_{\mathrm{d}}\left(I_{\mathrm{md}}, I_{\mathrm{mq}}\right)= & \\
= & \frac{\left(a_{\mathrm{d}_{10}}+a_{\mathrm{d}_{01}} I_{\mathrm{mq}}^{2}\right) I_{\mathrm{md}}+\left(a_{\mathrm{d}_{10}}+a_{\mathrm{d}_{11}} I_{\mathrm{mq}}^{2}\right) I_{\mathrm{md}}^{2}}{1+\left(b_{\mathrm{d}_{20}}+b_{\mathrm{d}_{21}} I_{\mathrm{mq}}^{2}\right) I_{\mathrm{md}}^{2}}
\end{aligned}
$$

— une partie linéaire $\lambda_{\mathrm{d}}=k_{2}+k_{3} \cdot I_{\mathrm{md}}$.

En se basant sur des données expérimentales, et en utilisant la méthode de Marquardt on a calculé les coefficients nécessaires pour les trois parties de l'ensemble des traces :

$$
\begin{aligned}
& a_{\mathrm{d}_{10}}=1,683 \\
& a_{\mathrm{d}_{11}}=-3,702 \times 10^{-3} \\
& a_{\mathrm{d}_{20}}=22,26 \\
& a_{\mathrm{d}_{21}}=-7,711 \times 10^{-3} \\
& b_{\mathrm{d}_{20}}=2,6136 \times 10^{-3} \\
& b_{\mathrm{d}_{21}}=-4,985 \times 10^{-7}
\end{aligned}
$$


avec :

$$
\begin{aligned}
& a_{\mathrm{d}_{1}}=a_{\mathrm{d}_{10}}+a_{\mathrm{d}_{11}} I_{\mathrm{mq}}^{2} \\
& a_{\mathrm{d}_{2}}=a_{\mathrm{d}_{20}}+a_{\mathrm{d}_{21}} I_{\mathrm{mq}}^{2} \\
& b_{\mathrm{d}_{2}}=b_{\mathrm{d}_{20}}+b_{\mathrm{d}_{21}} I_{\mathrm{mq}}^{2}
\end{aligned}
$$

et :

$I_{\mathrm{md}_{1}}=\frac{\left(-a_{\mathrm{d}_{2}} b_{\mathrm{d}_{2}}+\sqrt{a_{\mathrm{d}_{2}}^{2} b_{\mathrm{d}_{2}}^{2}+a_{\mathrm{d}_{1}}^{2} b_{\mathrm{d}_{2}}}\right)}{a_{\mathrm{d}_{1}} b_{\mathrm{d}_{2}}}$

$k_{1}=\frac{2 a_{\mathrm{d}_{1}} I_{\mathrm{md}_{1}}+a_{\mathrm{d}_{2}}-a_{\mathrm{d}_{2}} b_{\mathrm{d}_{2}} I_{\mathrm{md}}^{2}}{\left(1+b_{\mathrm{d}_{2}} I_{\mathrm{md}}^{2}\right)^{2}}$

$I_{\text {md }_{\max }}=0.9\left(\frac{a_{\mathrm{d}_{1}}+\sqrt{a_{\mathrm{d}_{1}}^{2}+a_{\mathrm{d}_{2}} b_{\mathrm{d}_{2}}}}{a_{\mathrm{d}_{2}} b_{\mathrm{d}_{2}}}\right)$

$k_{2}=\frac{a_{\mathrm{d}_{1}} I_{\mathrm{md}_{\max }}+a_{\mathrm{d}_{2}} I_{\mathrm{md}_{\max }}^{2}}{1+b_{\mathrm{d}_{2}} I_{\text {md }_{\max }}^{2}}$

$k_{3}=\frac{2 a_{\mathrm{d}_{1}} I_{\mathrm{md}_{\max }}+a_{\mathrm{d}_{2}}-a_{\mathrm{d}_{2}} b_{\mathrm{d}_{2}} I_{\mathrm{md}_{\max }}^{2}}{1+b_{\mathrm{d}_{2}} I_{\mathrm{md}_{\max }}^{2}}$

En ce qui concerne l'axe q, on peut procéder de la même manière en trois parties avec les formules (31) en calculant les valeurs des coefficients propres à l'axe q. En considérant l'allure réelle des courbes d'aimantation sur cet axe on a préféré une représentation plus simple de la forme (17) en une seule partie, avec :

$$
\lambda_{\mathrm{q}}\left(I_{\mathrm{md}}, I_{\mathrm{mq}}\right)=a\left(1-\mathrm{e}^{\frac{-\left|I_{\mathrm{mq}}\right|}{b_{0}+b_{1} I_{\mathrm{md}}^{2}}}\right)
$$

et :

$$
\begin{aligned}
& a=668,37 \\
& b_{0}=49,45 \\
& b_{1}=2,9837 .
\end{aligned}
$$

4. Exploitation des résultats dans la simulation d'un système machine-convertisseur.

Nous avons appliqué les résultats obtenus à la simulation du comportement d'une machine à réluctance variable mentionnée à 3.1.1. et alimentée par un convertisseur statique et autopilotée.

Un redresseur régulé fournit à partir d'un réseau $50 \mathrm{~Hz}$ une tension réglable. Celle-ci alimente la machine à travers un onduleur, dont les séquences de conduction sont commandées à partir d'un capteur de position lié au rotor.

Le schéma bloc de la figure 19 représente l'ensemble machine-convertisseur et son alimentation. Les figures 20 et 21 montrent respectivement l'évolution

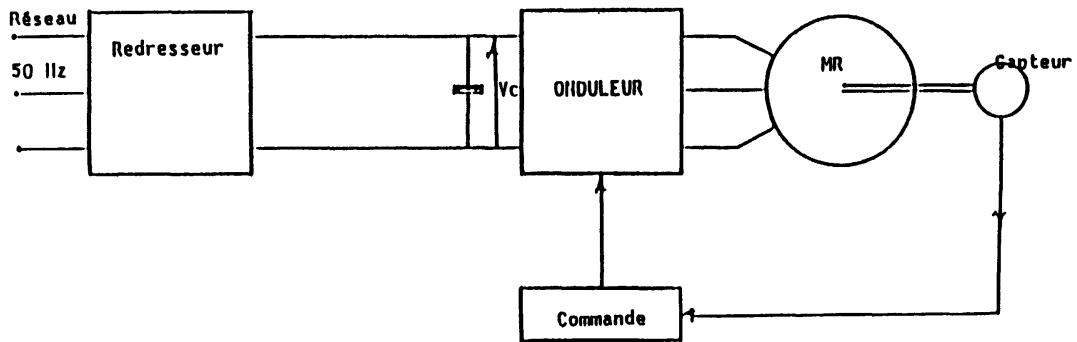

Fig. 19. - Schéma-bloc du système machine-convertisseur.

[Bloc diagram of converter-fed motor drive system.]

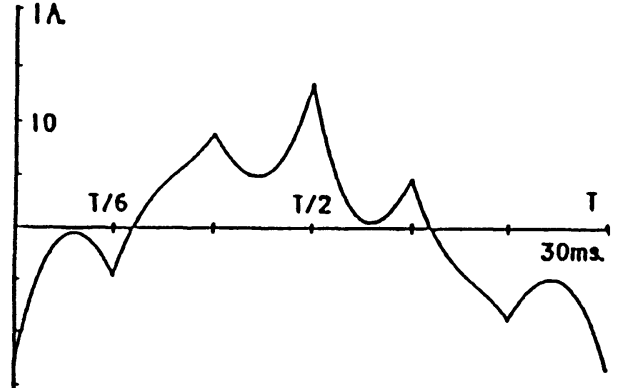

a)

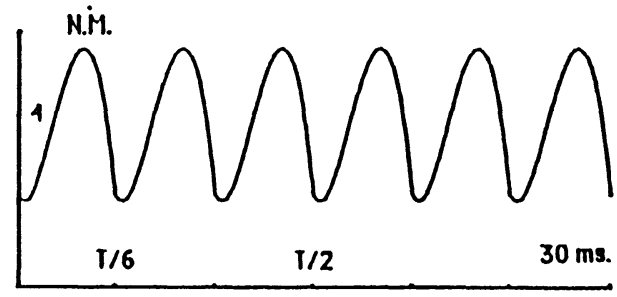

b)

Fig. 20. - Caractéristiques en régime permanent : a) courant de ligne ; b) couple électromagnétique.

[Steady-state characteristics $a$ of converter-fed motor drive system : a) line current ; b) electromagnetic torque.] 
a)

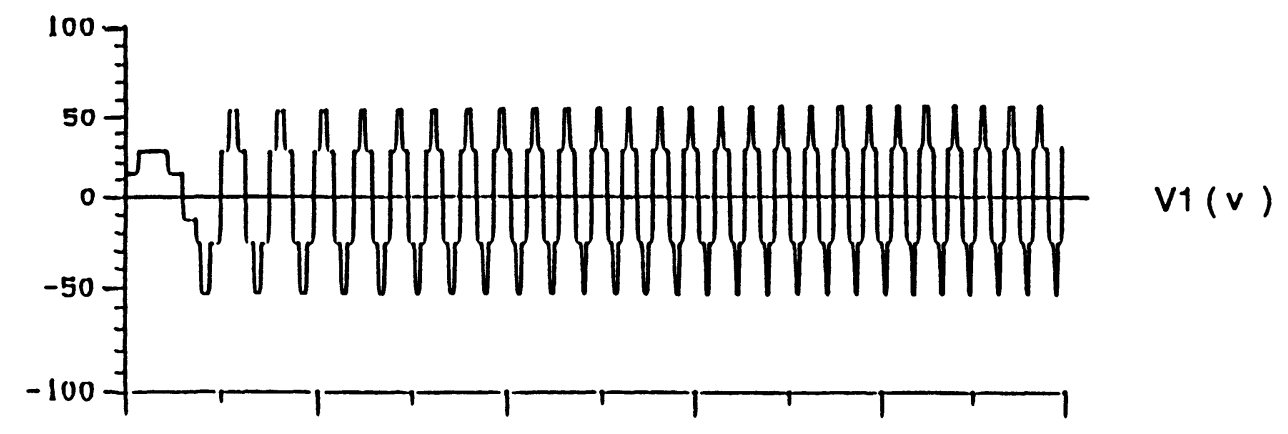

b)

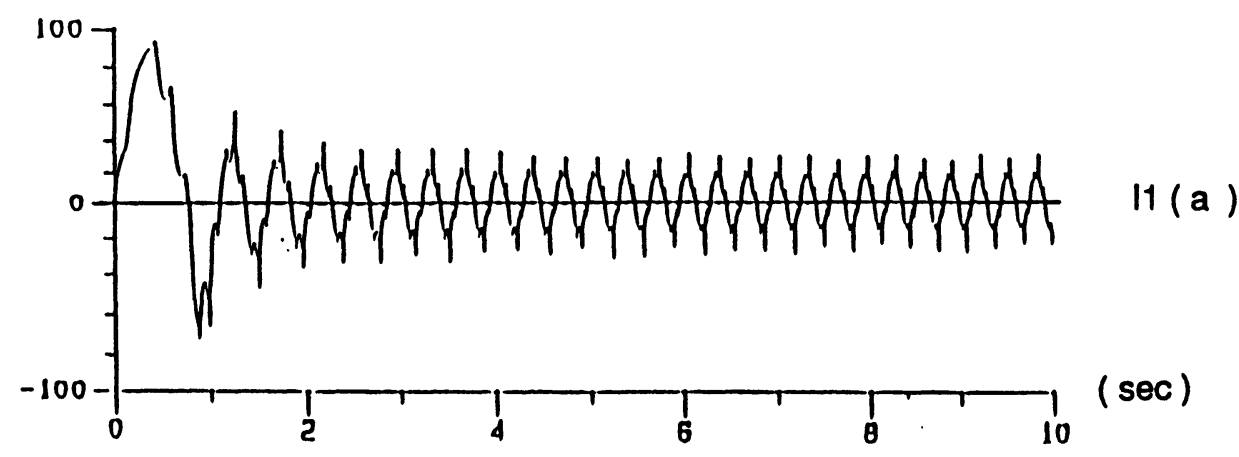

c)

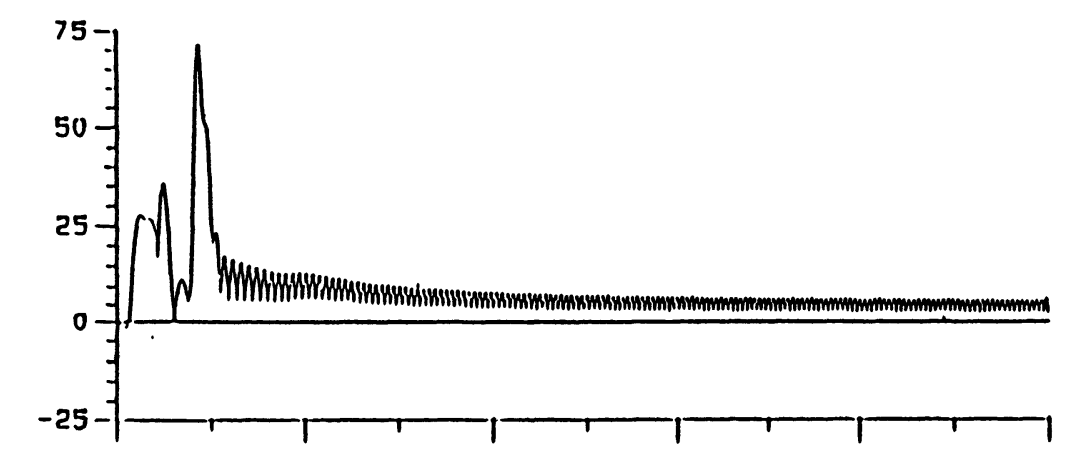

$\operatorname{Cem}(\mathrm{Nm})$

d)

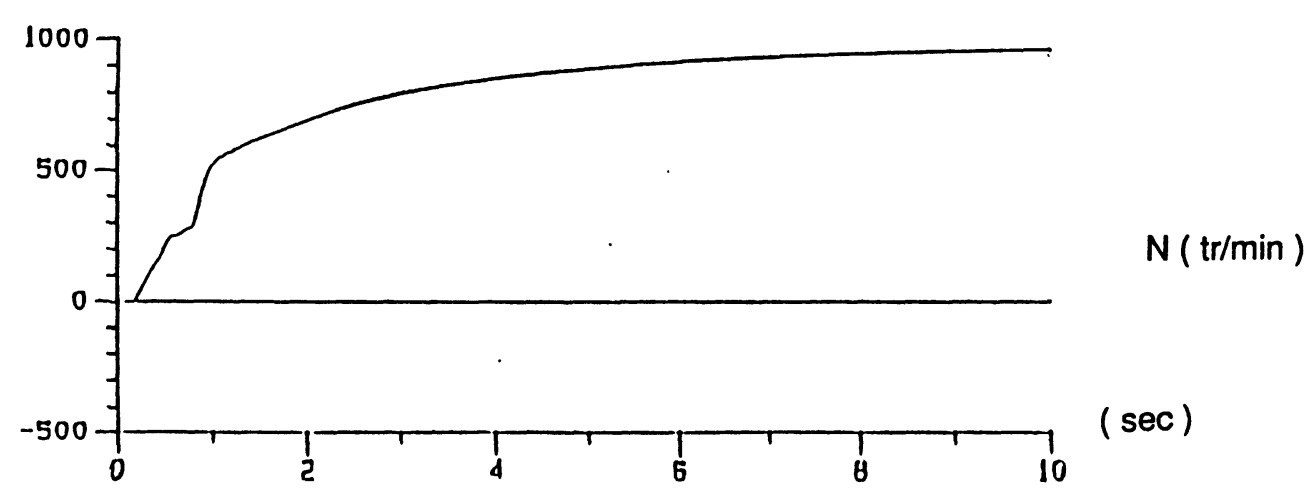

Fig. 21. - Caractéristiques en régime transitoire de démarrage : a) tension par phase ; b) courant de ligne ; c) couple électromagnétique ; d) vitesse.

[Transient-state characteristics during run-up period : a) per phase voltage ; b) line current ; c) electromagnetic torque ; d) speed.]

du courant et du couple pour une période de fonctionnement dans un enroulement statorique lors d'un régime permanent ainsi que la variation de la tension, du courant, du couple et de la vitesse de la machine lors d'un régime transitoire au démarrage du système.

\section{Conclusion.}

Une première étude de la modélisation des machines électriques à courant alternatif dans l'état de saturation en utilisant les surfaces d'aimantation a été mise en place. Connaissant analytiquement ces surfaces 
pour les machines à pôles saillants, on peut calculer à chaque point de fonctionnement, les valeurs des inductances statiques et dynamiques de la machine ainsi que les coefficients dus à l'effet croisé de saturation. Quant aux machines à pôles lisses on peut procéder de la même manière en notant que les surfaces d'aimantation sont alors identiques. Le modèle ainsi obtenu est un modèle qui répond correctement aux besoins des calculs précis liés à l'étude du comportement des machines, tant en régimes permanents que transitoires.

\section{Bibliographie}

[1] Maggetto G., Sneyers B., Van Eck J. L., Permanent magnet motor used for traction purposes, Motor Con. Proc. (Sept. 82).

[2] SNeyers B., Novotny D. W., Lipo T. A., Field weakening in buried permanent magnet A.c. motor drives, IEEE, IAS annual meeting (1984) pp. 462-468.

[3] Kovacs K. P., On the theory of cylindrical motor AC machine including main flux saturation, IEEE Trans. Power Apparatus Systems PAS103, $\mathrm{N}^{\circ} 4$ (april 1984).

[4] Brown J. E., Kovacs K. P., Vas P., A method of including the effect of main fux path saturation in the generalized equations of AC machines, IEEE Trans. Power Apparatus Systems PAS102, $\mathrm{N}^{\circ} 1$ (janv. 1983).

[5] Vas P., Brown J. E., Hallenius K. E., Crosssaturation effects in saturated smooth-air-gap machines, International Conference on Electrical Machines (1984) pp. 261, 264.

[6] Vas P., Hallenius K. E., Brown J. E., Crosssaturation in smooth-air-gap electrical machines, IEEE Trans. Energy Conversion EC-1 (1986) 103-109.

[7] Faucher J., Anvari H. A., Trannoy B., Modelling and simulation of a converter-fed synchronous motor drive with considering cross coupling saturation effect, IEEE-IAS 1984, Conf. Record, pp. 432-438.

[8] Anvari H. A., Faucher J., Trannoy B., Cross coupling saturation effect in synchronous machines and its comportation by finites differences solutions, Proc. ICEM, Int. Conf. on electric machines, part. 2 (sept. 1986) pp. 451-454, Munchen R.F.A.

[9] Anvari H. A., Faucher J., On the modelling of variable speed synchronous motor drives with considering cross magnetisation effect, Colloq.
International sur la modélisation et simulation des machines électriques, IMACS, IEEE, TC-1 Proc., Québec, Canada (1987) pp. 144-148.

[10] Anvari H. A., FAucher J., Simulation of some transient behaviours of a saturated converter-fed synchronous motor drive considering the crosscoupling effect, ICEM 1988 (Pisa, Italy) pp. 519524.

[11] ROBERT J., A simplified method for the study of saturation in AC machines 3, IMACS, IEEE, TC-1, Proc. , Canada (1987) pp. 117-124.

[12] Kamoun M., Poloujadoff M., Accounting of saturation and cross-saturation in a dynamic stability study, Electrical Power Syst. Res. 10 (1986) 5561.

[13] Kamoun M., Pillet E., Poloujadoff M., Prise en compte de la saturation dans le fonctionnement en régime établi de la machine synchrone, Rev. E. Soc. R. Belge Electr. 101 (1985) 5-9.

[14] Faucher J., Lajoie-Mazenc M., Chayeghani A., Characterisation of a closed-loop controlled current-fed reluctance machine taking into consideration saturation, IEEE-IAS Trans. 1A-15 (1979) 482-48.

[15] Adkins B., HARLEY R. G., The general theory of alternating current machines (Chapman and Hall London) pp. 213-217.

[16] Anvari H. A., Xhuvani A., Faucher J., Extended equivalent circuit model for simulation of transient and steady state behaviours of saturated converter-fed motor drive, AMSE International conference on modelling and simulation, Istambul, Turkey, vol. 2c (June 29-3 July 1988) p. 54, 62.

[17] WIDGER G. F. T., Representation of magnetisation curves over extensive range by rational-fraction approximation, Proc. IEEE $116 \mathrm{~N}^{\circ} 1$ (janv. 1969). 